\title{
Expanding the Substrate Scope for C-H Amination Reactions: Oxidative Cyclization of Urea and Guanidine Derivatives
}

Supplementary Material (43 pages)

Mihyong Kim, John V. Mulcahy, Christine G. Espino and J. Du Bois*

Department of Chemistry

Stanford University

Stanford, CA 94305-5080 
General. All reagents were obtained commercially unless otherwise noted. Reactions were performed using ovendried glassware under an atmosphere of dry nitrogen. Air- and moisture-sensitive liquids and solutions were transferred via syringe or stainless steel cannula. Organic solutions were concentrated under reduced pressure (ca. $15 \mathrm{~mm} \mathrm{Hg}$ ) by rotary evaporation. Solvents were purified by passage under $12 \mathrm{psi}_{2}$ through activated alumina columns. Chlorobenzene was purchased from Fisher Scientific and used without purification. Chlorosulfonyl isocyanate was obtained from Acros Chemicals, transferred via cannula to a Schlenk flask, and stored at $-20{ }^{\circ} \mathrm{C}$. 2,2,2-Trichloroethanol was purchased from Acros Chemicals and used as received. Light magnesium oxide was flame-dried under reduced pressure $(1 \mathrm{~mm} \mathrm{Hg})$ prior to use. The synthesis of 2,2,2-trichloroethylsulfamate was performed according to a previously published report. ${ }^{1}$ This material is also available commercially from Aldrich Chemical Co. Chromatographic purification of products was accomplished using forced-flow chromatography on EM Science Geduran silica gel $60(35-75 \mu \mathrm{m})$. Compounds purified by chromatography on silica gel were typically applied to the adsorbent bed using the indicated solvent conditions with a minimum amount of added dichloromethane as needed for solubility. Thin layer chromatography was performed on EM Science silica gel 60 $\mathrm{F}_{254}$ plates $(250 \mu \mathrm{m})$. Visualization of the developed chromatogram was accomplished by fluorescence quenching and by staining with ethanolic anisaldehyde, aqueous potassium permanganate, or aqueous ceric ammonium molybdate (CAM) solution.

Nuclear magnetic resonance (NMR) spectra were acquired on a Varian Mercury-400 operating at 400 and $100 \mathrm{MHz}$ or a Varian Inova-500 operating at 500 and $125 \mathrm{MHz}$ for ${ }^{1} \mathrm{H}$ and ${ }^{13} \mathrm{C}$, respectively, and are referenced internally according to residual solvent signals. Data for ${ }^{1} \mathrm{H}$ NMR are recorded as follows: chemical shift ( $\delta$, ppm), multiplicity (s, singlet; d, doublet, t, triplet; q, quartet; sept, septet; m, multiplet), integration, coupling constant (Hz). Data for ${ }^{13} \mathrm{C}$ are reported in terms of chemical shift $(\delta, \mathrm{ppm})$. Infrared spectra were recorded as thin films using $\mathrm{NaCl}$ salt plates on a Thermo-Nicolet IR300 spectrometer and are reported in frequency of absorption. Highresolution mass spectra were obtained from the Vincent Coates Foundation Mass Spectrometry Laboratory at Stanford University.

\section{Experimental Procedures and Characterization Data for Urea Substrates and Cyclic Products}

\section{$\mathrm{N}$-(2,2,2-Trichloroethoxysulfonyl)urea (TcesNHC(O) $\left.\mathrm{NH}_{2}\right)^{2}$}

Chlorosulfonyl isocyanate $(3.3 \mathrm{~mL}, 37.5 \mathrm{mmol})$ and $17 \mathrm{~mL}$ of $\mathrm{C}_{6} \mathrm{H}_{5} \mathrm{Cl}$ were combined in a $100 \mathrm{~mL}$ recovery flask equipped with a magnetic stir bar and a Liebig condenser. A solution of 2,2,2-trichloroethanol (3.6 mL, $37.5 \mathrm{mmol})$ in $25 \mathrm{~mL}$ of $\mathrm{C}_{6} \mathrm{H}_{5} \mathrm{Cl}$ was introduced dropwise via cannula over $5 \mathrm{~min}$. The rubber septum and $\mathrm{N}_{2}$ gas inlet atop the condenser were replaced with a drying tube containing $\mathrm{Na}_{2} \mathrm{CO}_{3}$ and the reaction was heated at reflux for $12 \mathrm{~h}$. Following this period, the reaction was cooled to $23{ }^{\circ} \mathrm{C}$ and the condenser was replaced by a short path distillation head equipped with a $100 \mathrm{~mL}$ receiving flask. The $\mathrm{C}_{6} \mathrm{H}_{5} \mathrm{Cl}$ solvent was removed by distillation to give a light brown viscous residue. This material was dissolved in $40 \mathrm{~mL}$ of $\mathrm{CH}_{2} \mathrm{Cl}_{2}$ and the solution was cooled to $0{ }^{\circ} \mathrm{C}$. Neat $\left(\mathrm{Me}_{3} \mathrm{Si}\right)_{2} \mathrm{NH}(7.9 \mathrm{~mL}, 37.5 \mathrm{mmol})$ was then added dropwise via syringe. The reaction mixture was stirred at $23{ }^{\circ} \mathrm{C}$ for $4 \mathrm{~h}$. The solution was then concentrated under reduced pressure to give an off-white solid, which was subsequently dissolved in $40 \mathrm{~mL}$ of $\mathrm{MeOH}$ and $300 \mu \mathrm{L}$ of AcOH. The resulting slurry was stirred vigorously for $5 \mathrm{~h}$ after which time all volatile materials were removed under reduced pressure and the oily product was dried azetropically with $50 \mathrm{~mL}$ of toluene. The solid mass was recrystallized from $\sim 300 \mathrm{~mL}$ of a 3:1 EtOAc/hexanes mixture to give the desired product as an amorphous white powder $(6.98 \mathrm{~g}, 69 \%)$. ${ }^{1} \mathrm{H} \mathrm{NMR}\left(\mathrm{CD}_{3} \mathrm{CN}, 400 \mathrm{MHz}\right) \delta$ 5.72 (br s, 2H), 4.87 (s, 2H) ppm; ${ }^{13} \mathrm{C}$ NMR $\left(\mathrm{CD}_{3} \mathrm{CN}, 100 \mathrm{MHz}\right) \delta 152.8,94.2,80.4 \mathrm{ppm}$; IR (thin film) v 3472, $1717,1374,1179,1007,914,873 \mathrm{~cm}^{-1}$. 


\section{Preparation of $\boldsymbol{N}$-Alkyl- $\boldsymbol{N}$-(2,2,2-trichloroethoxysulfonyl)urea Substrates}

To a mixture of alcohol $(7 \mathrm{mmol})$, TcesNHC(O)NH $\mathrm{NH}_{2}\left(2.53 \mathrm{~g}, 9.31 \mathrm{mmol}, 1.33\right.$ equiv), and $\mathrm{PPh}_{3}(2.39 \mathrm{~g}, 9.1 \mathrm{mmol}$, 1.3 equiv) in $23 \mathrm{~mL}$ of THF at $0{ }^{\circ} \mathrm{C}$ was added diethyl azodicarboxylate $(1.43 \mathrm{~mL}, 9.1 \mathrm{mmol}, 1.3$ equiv) dropwise via syringe. The solution was warmed to $23{ }^{\circ} \mathrm{C}$ and stirred for $4-6 \mathrm{~h}$ until TLC showed complete consumption of the starting alcohol. After this time, all volatile materials were removed under reduced pressure. The resulting oily material was suspended in $30 \mathrm{~mL}$ of hexanes and concentrated again. This process was repeated twice. The isolated product was purified by chromatography on silica gel (conditions given below).

\section{General Procedure for C-H Amination of Urea Substrates}

To a solution of urea substrate $(0.3 \mathrm{mmol})$ in $2.0 \mathrm{~mL}$ of toluene were added sequentially $\mathrm{MgO}(30 \mathrm{mg}, 0.75 \mathrm{mmol}$, 2.5 equiv), $\mathrm{PhI}(\mathrm{OAc})_{2}\left(145 \mathrm{mg}, 0.45 \mathrm{mmol}, 1.5\right.$ equiv) and $\mathrm{Rh}_{2}(\mathrm{esp})_{2}(2.3 \mathrm{mg}, 3 \mu \mathrm{mol}, 0.01$ equiv). The resulting green solution was stirred at $40{ }^{\circ} \mathrm{C}$ until TLC indicated no further progress in the reaction $(\sim 8 \mathrm{~h})$. The yellowish green reaction mixture was cooled to $23{ }^{\circ} \mathrm{C}$, diluted with $2 \mathrm{~mL}$ of $\mathrm{CH}_{2} \mathrm{Cl}_{2}$, and filtered through a pad of Celite $(20 \mathrm{x}$ $10 \mathrm{~mm}$ ). The filter cake was rinsed with $2 \times 3 \mathrm{~mL}$ of $\mathrm{CH}_{2} \mathrm{Cl}_{2}$ and the combined filtrates were evaporated under reduced pressure. Purification of the isolated material by chromatography on silica gel (conditions given below) afforded the desired product.

\section{Characterization Data for Urea Substrates and Corresponding Amination Products}

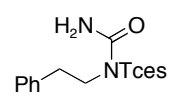

Purified by chromatography on silica gel (gradient elution: 6:1 $\rightarrow 4: 1$ hexanes/EtOAc); white solid (75\%): $\mathrm{TLC}_{f}=$ 0.4 (3:1 hexanes/EtOAc); ${ }^{1} \mathrm{H}$ NMR $\left(\mathrm{CDCl}_{3}, 400 \mathrm{MHz}\right) \delta$ 7.35-7.33 (m, 2H), 7.28-7.24 (m, 1H), 7.22-7.19 (m, 2H), 7.02 (br s, 1H), 5.49 (br s, 1H), $4.63(\mathrm{~s}, 2 \mathrm{H}), 4.48(\mathrm{t}, 2 \mathrm{H}, J=6.8 \mathrm{~Hz}), 3.00(\mathrm{t}, 2 \mathrm{H}, J=7.2 \mathrm{~Hz}) \mathrm{ppm} ;{ }^{13} \mathrm{C}$ NMR $\left(\mathrm{CDCl}_{3}, 100 \mathrm{MHz}\right) \delta 160.4,137.0,129.1,129.0,127.2,93.9,78.7,69.7,35.0 \mathrm{ppm}$; IR (thin film) v 3455, 3351, $1628,1544,1476,1447,1332,1177,1087,1017,853 \mathrm{~cm}^{-1}$; HRMS (ES ${ }^{-}$) calcd for $\mathrm{C}_{11} \mathrm{H}_{13} \mathrm{C}_{13} \mathrm{~N}_{2} \mathrm{O}_{4} \mathrm{~S} 373.9662$ found $372.9569\left(\mathrm{M}-\mathrm{H}^{+}\right)$.

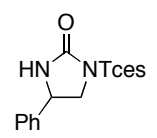

Purified by chromatography on silica gel (gradient elution: $5: 1 \rightarrow 2: 1$ hexanes/EtOAc); white solid (79\%): $\mathrm{TLC} \mathrm{R}_{f}=$ 0.25 (3:1 hexanes/EtOAc); ${ }^{1} \mathrm{H}$ NMR $\left(\mathrm{CDCl}_{3}, 400 \mathrm{MHz}\right) \delta 7.66$ (br s, $\left.1 \mathrm{H}\right), 7.47-7.41(\mathrm{~m}, 3 \mathrm{H}), 7.33-7.31(\mathrm{~m}, 2 \mathrm{H})$, $5.21(\mathrm{dd}, 1 \mathrm{H}, J=9.2,7.2 \mathrm{~Hz}), 4.94(\mathrm{t}, 1 \mathrm{H}, J=9.2 \mathrm{~Hz}), 4.73(\mathrm{~d}, 1 \mathrm{H}, J=10.8 \mathrm{~Hz}), 4.70(\mathrm{~d}, 1 \mathrm{H}, J=10.8 \mathrm{~Hz}), 4.42$ $(\mathrm{dd}, 1 \mathrm{H}, J=8.8,6.8 \mathrm{~Hz}) \mathrm{ppm} ;{ }^{13} \mathrm{C} \mathrm{NMR}\left(\mathrm{CDCl}_{3}, 100 \mathrm{MHz}\right) \delta 163.0,137.0,130.0,129.7,126.3,93.8,78.7,74.7$, $58.9 \mathrm{ppm}$; IR (thin film) v 3384, 1628, 1480, 1460, 1440, 1342, 1177, 1101, 1017, $879 \mathrm{~cm}^{-1}$; HRMS (ES ${ }^{-}$) calcd for $\mathrm{C}_{11} \mathrm{H}_{11} \mathrm{Cl}_{3} \mathrm{~N}_{2} \mathrm{O}_{4} \mathrm{~S} 371.9505$ found $370.9411\left(\mathrm{M}-\mathrm{H}^{+}\right)$.

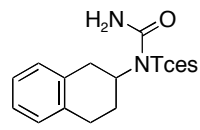

Purified by chromatography on silica gel (gradient elution: 6:1 $\rightarrow 3: 1$ hexanes/EtOAc); white solid (65\%): $\mathrm{TLC} \mathrm{R}_{f}=$ 0.3 (3:1 hexanes/EtOAc); ${ }^{1} \mathrm{H}$ NMR $\left(\mathrm{CDCl}_{3}, 400 \mathrm{MHz}\right) \delta$ 7.15-7.05 (m, 4H), 7.01 (br s, 1H), 5.56 (br s, $\left.1 \mathrm{H}\right), 5.40$ (tt, $1 \mathrm{H}, J=6.04 .8 \mathrm{~Hz}), 4.67(\mathrm{~s}, 2 \mathrm{H}), 3.12(\mathrm{dd}, 1 \mathrm{H}, J=16.8,4.8 \mathrm{~Hz}), 2.98-2.81(\mathrm{~m}, 3 \mathrm{H}), 2.08(\mathrm{td}, 2 \mathrm{H}, J=12.0,6.0 \mathrm{~Hz})$ ppm; ${ }^{13} \mathrm{C}$ NMR $\left(\mathrm{CDCl}_{3}, 100 \mathrm{MHz}\right) \delta 159.8,135.0,132.5,129.3,128.6,126.3,126.1,93.7,78.4,74.9,34.2,27.3$, $25.7 \mathrm{ppm}$; IR (thin film) v3456, 3351, 1628, 1542, 1452, 1336, 1178, 1091, 1018, $857 \mathrm{~cm}^{-1}$. 


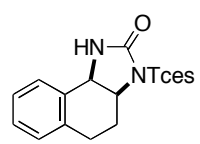

Purified by chromatography on silica gel (gradient elution: 8:1 $\rightarrow 4: 1$ hexanes/EtOAc); white solid (93\%): TLC $\mathrm{R}_{f}=$ 0.4 (3:1 hexanes/EtOAc); ${ }^{1} \mathrm{H}$ NMR $\left(\mathrm{CDCl}_{3}, 400 \mathrm{MHz}\right) \delta 7.43$ (br s, $\left.1 \mathrm{H}\right), 7.32-7.18(\mathrm{~m}, 4 \mathrm{H}), 5.37(\mathrm{td}, 1 \mathrm{H}, J=8.8$, $3.4 \mathrm{~Hz}), 5.23(\mathrm{~d}, 1 \mathrm{H}, J=8.8 \mathrm{~Hz}), 4.61(\mathrm{~s}, 2 \mathrm{H}), 2.85(\mathrm{ddd}, 1 \mathrm{H}, J=16.0,12.4,3.6 \mathrm{~Hz}), 2.69(\mathrm{dt}, 1 \mathrm{H}, J=16.0,4.2 \mathrm{~Hz})$, $2.42(\mathrm{dq}, 1 \mathrm{H}, J=14.8,3.4 \mathrm{~Hz}), 1.85(\mathrm{ddt}, 1 \mathrm{H}, J=14.8,12.0,4.0 \mathrm{~Hz}) \mathrm{ppm} ;{ }^{13} \mathrm{C} \mathrm{NMR}\left(\mathrm{CDCl}_{3}, 100 \mathrm{MHz}\right) \delta 162.3$, 138.0, 130.9, 129.6, 129.4, 129.3, 127.9, 93.9, 79.3, 78.8, 56.0, 27.6, 23.8 ppm; IR (thin film) v 3340, 1628, 1494, 1444, 1385, 1344, 1298, 1244, 1178, 1090, 1016, $853 \mathrm{~cm}^{-1}$; HRMS (ES ${ }^{+}$) calcd for $\mathrm{C}_{13} \mathrm{H}_{13} \mathrm{Cl}_{3} \mathrm{~N}_{2} \mathrm{O}_{4} \mathrm{~S} 397.9662$ found $398.9754\left(\mathrm{MH}^{+}\right)$.

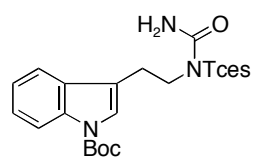

Purified by chromatography on silica gel (gradient elution: $6: 1 \rightarrow 3: 1$ hexanes/EtOAc); white solid (65\%): $\mathrm{TLC} \mathrm{R}_{f}=$ 0.3 (3:1 hexanes/EtOAc); ${ }^{1} \mathrm{H}$ NMR $\left(\mathrm{CDCl}_{3}, 400 \mathrm{MHz}\right) \delta 8.11$ (br d, $\left.1 \mathrm{H}, J=7.2 \mathrm{~Hz}\right), 7.50(\mathrm{ddd}, 1 \mathrm{H}, J=7.8,1.2,0.8$ $\mathrm{Hz}), 7.42$ (br s, 1H), 7.32 (ddd, $1 \mathrm{H}, J=8.4,7.6,1.2 \mathrm{~Hz}), 7.24$ (ddd, $1 \mathrm{H}, J=8.4,7.6,1.2 \mathrm{~Hz}), 7.02$ (br s, $1 \mathrm{H}), 5.47$ (br s, $1 \mathrm{H}), 4.62(\mathrm{~s}, 2 \mathrm{H}), 4.52(\mathrm{t}, 2 \mathrm{H}, J=6.8 \mathrm{~Hz}), 3.08(\mathrm{t}, 2 \mathrm{H}, J=6.8 \mathrm{~Hz}), 1.68(\mathrm{~s}, 9 \mathrm{H}) \mathrm{ppm} ;{ }^{13} \mathrm{C} \mathrm{NMR}\left(\mathrm{CDCl}_{3}, 100\right.$ MHz) $\delta 160.2,149.6,135.3,130.1,124.6,123.5,122.6,118.6,115.6,115.4,93.6,83.8,78.4,68.2,28.2,24.3$ ppm; IR (thin film) v 3451, 3341, 1730, 1633, 1549, 1452, 1372, 1258, 1160, 1092, 1019, $853 \mathrm{~cm}^{-1}$.

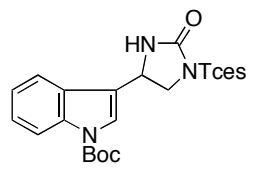

Purified by chromatography on silica gel (gradient elution: 3:1 $\rightarrow 1: 1$ hexanes/EtOAc); off-white solid (73\%): TLC $\mathrm{R}_{f}=0.2\left(3: 1\right.$ hexanes/EtOAc); ${ }^{1} \mathrm{H} \mathrm{NMR}\left(\mathrm{CDCl}_{3}, 400 \mathrm{MHz}\right) \delta 8.17(\mathrm{~d}, 1 \mathrm{H}, J=4.2 \mathrm{~Hz}), 7.64(\mathrm{~s}, 1 \mathrm{H}), 7.45(\mathrm{ddd}, 1 \mathrm{H}, J$ $=7.8,1.2,0.8 \mathrm{~Hz}), 7.37(\mathrm{ddd}, 1 \mathrm{H}, J=8.4,7.2,1.2 \mathrm{~Hz}), 7.25(\mathrm{td}, 1 \mathrm{H}, J=7.6,1.2 \mathrm{~Hz}), 5.45(\mathrm{dd}, 1 \mathrm{H}, J=9.6,6.8 \mathrm{~Hz})$, $4.93(\mathrm{t}, 1 \mathrm{H}, J=9.2 \mathrm{~Hz}), 4.65(\mathrm{~s}, 2 \mathrm{H}), 4.62(\mathrm{dd}, 1 \mathrm{H}, J=9.2,6.8 \mathrm{~Hz}), 1.65(\mathrm{~s}, 9 \mathrm{H}) \mathrm{ppm} ;{ }^{13} \mathrm{C} \mathrm{NMR}\left(\mathrm{CDCl}_{3}, 100 \mathrm{MHz}\right)$ ठ 162.6, 149.1, 136.1, 126.6, 125.5, 124.7, 123.4, 118.4, 115.96, 115.93, 93.6, 84.7, 78.6, 72.4, 52.2, 28.1 ppm; IR

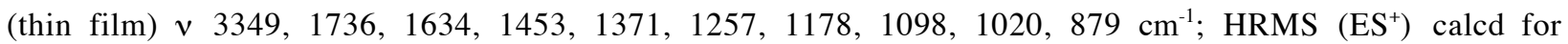
$\mathrm{C}_{18} \mathrm{H}_{20} \mathrm{Cl}_{3} \mathrm{~N}_{3} \mathrm{O}_{6} \mathrm{~S} 511.0138$ found $534.0032\left(\mathrm{MNa}^{+}\right)$.

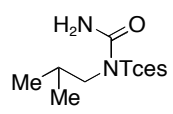

Purified by chromatography on silica gel (gradient elution: $8: 1 \rightarrow 3: 1$ hexanes/EtOAc); white solid (75\%): $\mathrm{TLC} \mathrm{R}_{f}=$ 0.4 (3:1 hexanes/EtOAc); ${ }^{1} \mathrm{H}$ NMR $\left(\mathrm{CDCl}_{3}, 400 \mathrm{MHz}\right) \delta 7.02$ (br s, 1H), 5.61 (br s, 1H), 4.65 (s, 2H), 4.02 (d, $2 \mathrm{H}, J$ $=6.4 \mathrm{~Hz}), 2.03-1.93(\mathrm{~m}, 1 \mathrm{H}), 0.94(\mathrm{~d}, 6 \mathrm{H}, J=6.4 \mathrm{~Hz}) \mathrm{ppm} ;{ }^{13} \mathrm{C} \mathrm{NMR}\left(\mathrm{CDCl}_{3}, 100 \mathrm{MHz}\right) \delta 160.5,93.7,78.4,75.2$, 27.5, 18.8 ppm; IR (thin film) v 3477, 3371, 1615, 1543, 1442, 1323, 1170, 1087, 1013, $874 \mathrm{~cm}^{-1}$; $\mathrm{HRMS}_{(\mathrm{ES}}^{+}$) calcd for $\mathrm{C}_{7} \mathrm{H}_{13} \mathrm{Cl}_{3} \mathrm{~N}_{2} \mathrm{O}_{4} \mathrm{~S} 325.9662$ found $\left(\mathrm{M}-\mathrm{H}^{+}\right)$.

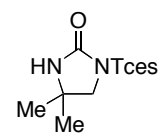

Purified by chromatography on silica gel (gradient elution: 6:1 $\rightarrow 3: 1$ hexanes/EtOAc); white solid (92\%): mp 127-130 ${ }^{\circ} \mathrm{C}$; TLC $\mathrm{R}_{f}=0.3$ (3:1 hexanes/EtOAc); ${ }^{1} \mathrm{H}$ NMR $\left(\mathrm{CDCl}_{3}, 400 \mathrm{MHz}\right) \delta 7.76$ (br s, $\left.1 \mathrm{H}\right), 4.64(\mathrm{~s}, 2 \mathrm{H}), 4.26$ (s, 2H), $1.46(\mathrm{~s}, 6 \mathrm{H}) \mathrm{ppm} ;{ }^{13} \mathrm{C} \mathrm{NMR}\left(\mathrm{CDCl}_{3}, 100 \mathrm{MHz}\right) \delta 161.6,93.7,79.2,78.5,59.1,27.0 \mathrm{ppm}$; IR (thin film) $v$ 
$3341,1631,1443,1343,1171,1090,1020,882 \mathrm{~cm}^{-1}$; HRMS $\left(\mathrm{ES}^{+}\right)$calcd for $\mathrm{C}_{7} \mathrm{H}_{11} \mathrm{Cl}_{3} \mathrm{~N}_{2} \mathrm{O}_{4} \mathrm{~S} 323.9505$ found $346.9390\left(\mathrm{MNa}^{+}\right)$.

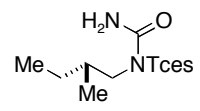

Purified by chromatography on silica gel (gradient elution: $8: 1 \rightarrow 3: 1$ hexanes/EtOAc); white solid (70\%): TLC $_{f}=$ 0.4 (3:1 hexanes/EtOAc); ${ }^{1} \mathrm{H}$ NMR $\left(\mathrm{CDCl}_{3}, 400 \mathrm{MHz}\right) \delta 7.02$ (br s, $\left.1 \mathrm{H}\right), 5.48(\mathrm{br} \mathrm{s}, 1 \mathrm{H}), 4.65(\mathrm{~s}, 2 \mathrm{H}), 4.11(\mathrm{dd}, 1 \mathrm{H}$, $J=10.4,4.4 \mathrm{~Hz}) 4.03(\mathrm{dd}, 1 \mathrm{H}, J=10.8,4.6 \mathrm{~Hz}), 1.77-1.71(\mathrm{~m}, 1 \mathrm{H}), 1.36-1.43(\mathrm{~m}, 1 \mathrm{H}), 1.24-1.13(\mathrm{~m}, 1 \mathrm{H}), 0.93-$ $0.88(\mathrm{~m}, 6 \mathrm{H}) \mathrm{ppm} ;{ }^{13} \mathrm{C} \mathrm{NMR}\left(\mathrm{CDCl}_{3}, 100 \mathrm{MHz}\right) \delta 160.6,93.7,78.5,73.9,34.0,25.7,16.1,11.1 \mathrm{ppm}$; IR (thin film) $v 3478,3368,1614,1543,1453,1323,1171,1088,1012,877 \mathrm{~cm}^{-1}$.

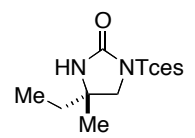

Purified by chromatography on silica gel (gradient elution: $6: 1 \rightarrow 3: 1$ hexanes/EtOAc); white solid (84\%): $\mathrm{TLC} \mathrm{R}_{f}=$ 0.3 (3:1 hexanes/EtOAc); ${ }^{1} \mathrm{H}$ NMR $\left(\mathrm{CDCl}_{3}, 400 \mathrm{MHz}\right) \delta 7.50$ (br s, 1H), 4.65 (s, 2H), 4.33 (d, 1H, $\left.J=8.8 \mathrm{~Hz}\right), 4.20$ $(\mathrm{d}, 1 \mathrm{H}, J=8.8 \mathrm{~Hz}), 1.76-1.65(\mathrm{~m}, 2 \mathrm{H}), 1.43(\mathrm{~s}, 3 \mathrm{H}), 0.95(\mathrm{t}, 3 \mathrm{H}, J=7.4 \mathrm{~Hz}) \mathrm{ppm} ;{ }^{13} \mathrm{C} \mathrm{NMR}\left(\mathrm{CDCl}_{3}, 100 \mathrm{MHz}\right) \delta$ 162.0, 93.7, 78.5, 77.1, 62.1, 32.6, 25.1, 8.0 ppm; IR (thin film) v 3477, 3371, 1615, 1543, 1455, 1323, 1170, 1087, $1013,874 \mathrm{~cm}^{-1}$; HRMS $\left(\mathrm{ES}^{+}\right)$calcd for $\mathrm{C}_{8} \mathrm{H}_{13} \mathrm{Cl}_{3} \mathrm{~N}_{2} \mathrm{O}_{4} \mathrm{~S} 337.9662$ found $360.9718\left(\mathrm{MNa}^{+}\right)$.<smiles>C=CCCN(C)C(N)=O</smiles>

Purified by chromatography on silica gel (gradient elution: 8:1 $\rightarrow 4: 1$ hexanes/EtOAc); white crystalline solid (60\%): TLC $\mathrm{R}_{f}=0.4$ (3:1 hexanes/EtOAc); ${ }^{1} \mathrm{H}$ NMR $\left(\mathrm{CDCl}_{3}, 400 \mathrm{MHz}\right) \delta 7.02(\mathrm{br} \mathrm{s}, 1 \mathrm{H}), 5.74$ (ddt, $1 \mathrm{H}, J=17.2,10.4,6.8$ $\mathrm{Hz}), 5.50(\mathrm{br} \mathrm{s}, 1 \mathrm{H}), 5.15-5.08(\mathrm{~m}, 2 \mathrm{H}), 4.65(\mathrm{~s}, 2 \mathrm{H}), 4.29(\mathrm{t}, 2 \mathrm{H}, J=6.6 \mathrm{~Hz}), 2.47-2.42(\mathrm{~m}, 2 \mathrm{H}) \mathrm{ppm} ;{ }^{13} \mathrm{C}$ NMR $\left(\mathrm{CDCl}_{3}, 100 \mathrm{MHz}\right) \delta 160.3,133.0,118.1,93.6,78.5,68.2,32.7 \mathrm{ppm}$; IR (thin film) v3479, 3372, 1616, 1544, 1460, $1324,1171,1014,876 \mathrm{~cm}^{-1}$.

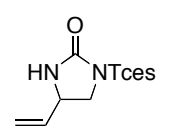

Purified by chromatography on silica gel (gradient elution: $6: 1 \rightarrow 3: 1$ hexanes/EtOAc); white solid $(41 \%)$ : TLC $\mathrm{R}_{f}=$ 0.35 (3:1 hexanes/EtOAc); ${ }^{1} \mathrm{H}$ NMR $\left(\mathrm{CDCl}_{3}, 400 \mathrm{MHz}\right) \delta 7.42$ (br s, $\left.1 \mathrm{H}\right), 5.81$ (ddd, $1 \mathrm{H}, J=17.2,10.0,7.2 \mathrm{~Hz}$ ), $5.42(\mathrm{~d}, 1 \mathrm{H}, J=16.8 \mathrm{~Hz}), 5.38(\mathrm{~d}, 1 \mathrm{H}, J=10.4 \mathrm{~Hz}), 4.72(\mathrm{t}, 1 \mathrm{H}, J=8.8 \mathrm{~Hz}), 4.67-4.61(\mathrm{~m}, 1 \mathrm{H}), 4.67(\mathrm{~s}, 2 \mathrm{H}), 4.26$ $(\mathrm{dd}, 1 \mathrm{H}, J=8.6,7.6 \mathrm{~Hz}), \mathrm{ppm} ;{ }^{13} \mathrm{C} \mathrm{NMR}\left(\mathrm{CDCl}_{3}, 100 \mathrm{MHz}\right) \delta 162.6,133.2,120.9,93.6,78.6,72.1,57.7 \mathrm{ppm}$; IR (thin film) $v 3350,1629,1444,1344,1179,1019,881 \mathrm{~cm}^{-1}$; HRMS $\left(\right.$ ES $^{+}$) calcd for $\mathrm{C}_{7} \mathrm{H}_{9} \mathrm{Cl}_{3} \mathrm{~N}_{3} \mathrm{O}_{4} \mathrm{~S} 321.9349$ found $344.9237\left(\mathrm{MNa}^{+}\right)$.

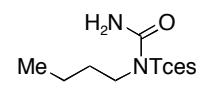

Purified by chromatography on silica gel (gradient elution: $8: 1 \rightarrow 4: 1$ hexanes/EtOAc); white, crystalline solid (70\%): TLC $\mathrm{R}_{f}=0.3\left(3: 1\right.$ hexanes/EtOAc); ${ }^{1} \mathrm{H} \mathrm{NMR}\left(\mathrm{CDCl}_{3}, 400 \mathrm{MHz}\right) \delta 7.01$ (br s, 1H), 5.46 (br s, 1H), 4.65 (s, $2 \mathrm{H}), 4.23(\mathrm{t}, 2 \mathrm{H}, J=6.6 \mathrm{~Hz}), 1.68-1.61(\mathrm{~m}, 2 \mathrm{H}), 1.42-1.33(\mathrm{~m}, 2 \mathrm{H}), 0.92(\mathrm{t}, 3 \mathrm{H}, J=7.4 \mathrm{~Hz}) \mathrm{ppm} ;{ }^{13} \mathrm{C} \mathrm{NMR}\left(\mathrm{CDCl}_{3}\right.$, $100 \mathrm{MHz}) \delta 160.4,93.7,78.5,69.3,30.3,18.8,13.6 \mathrm{ppm}$; IR (thin film) v3474, 3366, 1616, 1543, 1322, 1171, $1013,876 \mathrm{~cm}^{-1}$. 


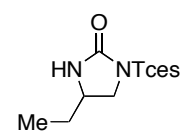

Purified by chromatography on silica gel (gradient elution: $4: 1 \rightarrow 2: 1$ hexanes/EtOAc); white solid (31\%): $\mathrm{TLC} \mathrm{R}_{f}=$ 0.2 (3:1 hexanes/EtOAc); ${ }^{1} \mathrm{H}$ NMR $\left(\mathrm{CDCl}_{3}, 400 \mathrm{MHz}\right) \delta 7.54(\mathrm{br} \mathrm{s}, 1 \mathrm{H}), 4.65(\mathrm{t}, 1 \mathrm{H}, J=8.8 \mathrm{~Hz}), 4.65$ (s, $\left.2 \mathrm{H}\right), 4.23$ $(\mathrm{dd}, 1 \mathrm{H}, J=8.8,6.4 \mathrm{~Hz}), 4.16-4.10(\mathrm{~m}, 1 \mathrm{H}), 1.77-1.62(\mathrm{~m}, 2 \mathrm{H}), 0.96(\mathrm{t}, 3 \mathrm{H}, J=7.4 \mathrm{~Hz}) \mathrm{ppm} ;{ }^{13} \mathrm{C}$ NMR $\left(\mathrm{CDCl}_{3}, 100\right.$ MHz) $\delta$ 162.7, 93.7, 78.5, 72.1, 56.4, 27.3, 9.0 ppm; IR (thin film) v 3356, 1633, 1445, 1346, 1179, 1089, 1020, 883 $\mathrm{cm}^{-1}$; HRMS (ES $)$ calcd for $\mathrm{C}_{7} \mathrm{H}_{11} \mathrm{Cl}_{3} \mathrm{~N}_{3} \mathrm{O}_{4} \mathrm{~S} 321.9349$ found $346.9398\left(\mathrm{MNa}^{+}\right)$.

\section{Experimental Procedures and Characterization Data for Guanidine Substrates and Cyclic Products}

\section{Preparation of $S, S$-Dimethyl- $N$-(2,2,2-trichloroethoxysulfonyl)carbonimidodithionate (TcesN=C(SMe) $\left.)_{2}\right)$} To a stirred solution of 2,2,2-trichloroethylsulfamate $(6.85 \mathrm{~g}, 30.0 \mathrm{mmol})$ in $23.0 \mathrm{~mL}$ of DMF at $0{ }^{\circ} \mathrm{C}$ were added sequentially $20 \mathrm{M}$ aqueous $\mathrm{NaOH}\left(2.10 \mathrm{~mL}, 42.0 \mathrm{mmol}, 1.4\right.$ equiv) and $\mathrm{CS}_{2}(1.07 \mathrm{~mL}, 17.8 \mathrm{mmol}, 0.6$ equiv). Two additional portions of $20 \mathrm{M}$ aqueous $\mathrm{NaOH}\left(1.05 \mathrm{~mL}, 21.0 \mathrm{mmol}, 0.7\right.$ equiv) and $\mathrm{CS}_{2}(0.54 \mathrm{~mL}, 9.0 \mathrm{mmol}, 0.3$ equiv) were added at $15 \mathrm{~min}$ intervals. The contents were stirred at $0{ }^{\circ} \mathrm{C}$ for $20 \mathrm{~min}$ and then warmed to $23{ }^{\circ} \mathrm{C}$. After $2 \mathrm{~h}$ the reddish-orange solution was cooled to $0{ }^{\circ} \mathrm{C}$ and $\mathrm{Me}_{2} \mathrm{SO}_{4}(5.97 \mathrm{~mL}, 63 \mathrm{mmol}, 2.1$ equiv) was added dropwise over $10 \mathrm{~min}$. The resulting yellow solution was warmed to $23{ }^{\circ} \mathrm{C}$ and stirred for $3 \mathrm{~h}$ after which time 40 $\mathrm{mL}$ of $\mathrm{H}_{2} \mathrm{O}$ was added slowly. The mixture was cooled once again to $0{ }^{\circ} \mathrm{C}$ and stirred vigorously. A yellow-orange solid slowly precipitated $(\sim 1 \mathrm{~h})$, which was collected upon filtration through a fritted funnel. Recrystallization of this material from $\sim 75 \mathrm{~mL}$ of $\mathrm{MeOH}$ ( 2 crops) afforded the desired product as white needles $(5.50 \mathrm{~g}, 55 \%)$ : TLC $\mathrm{R}_{f}$ $=0.45$ (2:1 hexanes/EtOAc); mp 61-62 ${ }^{\circ} \mathrm{C} ;{ }^{1} \mathrm{H}$ NMR $\left(\mathrm{CDCl}_{3}, 400 \mathrm{MHz}\right) \delta 4.71(\mathrm{~s}, 2 \mathrm{H}), 2.60(\mathrm{~s}, 6 \mathrm{H}) \mathrm{ppm} ;{ }^{13} \mathrm{C} \mathrm{NMR}$ $\left(\mathrm{CDCl}_{3}, 100 \mathrm{MHz}\right) \delta 190.0,93.3,79.3,16.7 \mathrm{ppm}$; IR (thin film) v 3024, 2958, 1425, 1357, 1267, 1171, 1090, 1012, $840 \mathrm{~cm}^{-1}$.

\section{Preparation of $S$-Methyl- $N$-(2,2,2-trichloroethoxysulfonyl)isothiourea (TcesN=C(SMe) $\mathbf{N H}_{2}$ )}

To a suspension of $S, S$-dimethyl- $N$-(2,2,2-trichloroethoxysulfonyl)carbonimidodithionate (3.26 g, $9.79 \mathrm{mmol})$ in 10 $\mathrm{mL}$ of $\mathrm{MeOH}$ was added $2 \mathrm{M}$ methanolic $\mathrm{NH}_{3}(5.38 \mathrm{~mL}, 10.8 \mathrm{mmol}, 1.1$ equiv). The reaction mixture was heated to $50{ }^{\circ} \mathrm{C}$ and stirred for $1 \mathrm{~h}$. The solution was cooled to $23^{\circ} \mathrm{C}$ and concentrated under reduced pressure. Purification of the oily residue by chromatography on silica gel (gradient elution: 4:1 $\rightarrow 2: 1$ hexanes/EtOAc) afforded the desired product as a white solid $(2.48 \mathrm{~g}, 84 \%)$ : TLC $\mathrm{R}_{f}=0.33$ (2:1 hexanes/EtOAc); mp 64-65 ${ }^{\circ} \mathrm{C} ;{ }^{1} \mathrm{H}$ NMR $\left(\mathrm{CDCl}_{3}, 400\right.$ $\mathrm{MHz}) \delta 4.66$ (s, 2H), 2.45 (s, 3H) ppm; ${ }^{13} \mathrm{C} \mathrm{NMR}\left(\mathrm{CDCl}_{3}, 100 \mathrm{MHz}\right) \delta 171.9,93.6,78.8,14.3 \mathrm{ppm}$; IR (KBr pellet) $v 3451,3343,1608,1512,1360,1158,1010,883,829,785 \mathrm{~cm}^{-1}$.

\section{Preparation of (2,2,2-Trichloroethoxysulfonyl)carbonchloroimidothioic acid methyl ester (TcesN=C(SMe)Cl)}

Sulfuryl chloride ( $903 \mu \mathrm{L}, 11.2 \mathrm{mmol}, 2.5$ equiv) was added dropwise to a stirred solution of $S, S$-dimethyl- $N$-(2,2,2trichloroethoxysulfonyl)carbonimidodithionate $(1.5 \mathrm{~g}, 4.51 \mathrm{mmol})$ in $4.5 \mathrm{~mL}$ of $\mathrm{CH}_{2} \mathrm{Cl}_{2}$. After $6 \mathrm{~h}$ the reaction was concentrated in vacuo $(\sim 1$ torr) to a slightly yellow solid. Purification by chromatography on silica gel (gradient elution: 9:1 $\rightarrow 5: 1$ hexanes/EtOAc) afforded the desired compound as a white solid $(1.36 \mathrm{~g}, 94 \%): \mathrm{mp} 61-64{ }^{\circ} \mathrm{C} ;{ }^{1} \mathrm{H}$ NMR $\left(\mathrm{CDCl}_{3}, 500 \mathrm{MHz}\right) \delta 4.76(\mathrm{~s}, 2 \mathrm{H}), 2.54(\mathrm{~s}, 3 \mathrm{H}) \mathrm{ppm} ;{ }^{13} \mathrm{C} \mathrm{NMR}\left(\mathrm{CDCl}_{3}, 100 \mathrm{MHz}\right) \delta 166.5,92.8,79.6,18.4$ ppm; IR (thin film) $v 1561,1372,1181,1086,1003,951,872,835 \mathrm{~cm}^{-1}$. 


\section{Preparation of $N$-Alkyl- $N$-(2,2,2-trichloroethoxysulfonyl)guanidines (Tces guanidine)}

\section{Method A}

A $15 \mathrm{~mL}$ thick-walled tube was charged with $S$-methyl- $N$-(2,2,2-trichloroethoxysulfonyl)isothiourea (754 mg, 2.5 mmol), $1^{\circ}$ amine ( $\left.2.5 \mathrm{mmol}\right), 2.5 \mathrm{~mL}$ of $\mathrm{H}_{2} \mathrm{O}$, and a magnetic stir bar. The vessel was sealed with a teflon screw-cap and the contents heated at $100{ }^{\circ} \mathrm{C}$ for $4 \mathrm{~h}$. The reaction mixture was cooled to $23{ }^{\circ} \mathrm{C}$ and $5 \mathrm{~mL}$ of $\mathrm{CH}_{2} \mathrm{Cl}_{2}$ was added. The biphasic solution was transferred to a separatory funnel, partitioned between $10 \mathrm{~mL} \mathrm{CH}_{2} \mathrm{Cl}_{2}$ and $10 \mathrm{~mL} \mathrm{H}_{2} \mathrm{O}$, and extracted with $3 \times 10 \mathrm{~mL}$ of $\mathrm{CH}_{2} \mathrm{Cl}_{2}$. The combined organic extracts were dried over $\mathrm{MgSO}_{4}$ and concentrated under reduced pressure. Purification of the isolated material by chromatography on silica gel (conditions given below) afforded the desired guanidine substrate.

\section{Method B}

A solution of (2,2,2-trichloroethoxysulfonyl)carbonchloroimidothioic acid methyl ester (353 mg, $1.1 \mathrm{mmol}, 1.1$ equiv) in $5 \mathrm{~mL}$ of $\mathrm{CH}_{2} \mathrm{Cl}_{2}$ was cooled to $0{ }^{\circ} \mathrm{C}$ and neat $1^{\circ}$ amine $(1 \mathrm{mmol})$ was added dropwise. To the resulting slightly turbid mixture was added triethylamine $\left(182 \mu \mathrm{L}, 1.2 \mathrm{mmol}, 1.2\right.$ equiv). The reaction was warmed to $23^{\circ} \mathrm{C}$ and stirred for $4 \mathrm{~h}$. The pale yellow solution was then concentrated in vacuo. Purification by chromatography on silica gel (conditions given below) furnished the isothiourea product.

To a solution of isothiourea $(0.5 \mathrm{mmol})$ in $5 \mathrm{~mL}$ of $\mathrm{MeCN}$ was added successively $\left(\mathrm{Me}_{3} \mathrm{Si}\right){ }_{2} \mathrm{NH}(265 \mu \mathrm{L}, 1.25 \mathrm{mmol}$, 2.5 equiv) and $\mathrm{HgCl}_{2}(149 \mathrm{mg}, 0.55 \mathrm{mmol}, 1.1$ equiv). After $1 \mathrm{~h}$ the milky white suspension was filtered through Celite and the filtrate was concentrated under reduced pressure. Purification of the oily residue by chromatography on silica gel (conditions given below) gave the desired $N$-Tces guanidine.

\section{General Procedure for C-H Amination of Guanidine Substrates}

A flame-dried $25 \mathrm{~mL}$ Schlenk flask was charged with Tces guanidine $(0.2 \mathrm{mmol}), \mathrm{Rh}_{2}(\mathrm{esp})_{2}(3 \mathrm{mg}, 4 \mu \mathrm{mol}, 0.02$ equiv), $\mathrm{PhI}(\mathrm{OAc})_{2}(106 \mathrm{mg}, 0.33 \mathrm{mmol}, 1.65$ equiv), and $\mathrm{MgO}(20 \mathrm{mg}, 0.5 \mathrm{mmol}, 2.5$ equiv). The reaction vessel was stoppered with a rubber septum, the contents placed briefly under vacuum $(\sim 5$ torr $)$, and the flask then backfilled with $\mathrm{N}_{2}$. This process was repeated two additional times prior to the addition of $2 \mathrm{~mL}$ of deoxygenated toluene. The resulting deep green suspension was heated to $40{ }^{\circ} \mathrm{C}$ and stirred for $12 \mathrm{~h}$. After this time, the mixture was cooled to $23{ }^{\circ} \mathrm{C}$, applied directly to a column of silica gel, and the product eluted using the conditions given below.

\section{Characterization Data for Guanidine Substrates and Corresponding Amination Products}

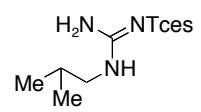

Prepared according to method A. Purified by chromatography on silica gel (gradient elution: $3: 1 \rightarrow 1: 1$ hexanes/EtOAc); white solid (80\%): mp 96-97 ${ }^{\circ} \mathrm{C}$; TLC $\mathrm{R}_{f}=0.22\left(1: 1\right.$ toluene/Et $\left.{ }_{2} \mathrm{O}\right) ;{ }^{1} \mathrm{H} \mathrm{NMR}\left(\mathrm{CD}_{3} \mathrm{CN}, 400 \mathrm{MHz}\right.$, $\left.60{ }^{\circ} \mathrm{C}\right) \delta 6.11(\mathrm{br} \mathrm{s}, 3 \mathrm{H}), 4.59(\mathrm{~s}, 2 \mathrm{H}), 3.02(\mathrm{t}, 2 \mathrm{H}, J=6.2 \mathrm{~Hz}), 1.83(\mathrm{sept}, 1 \mathrm{H}, J=6.8 \mathrm{~Hz}), 0.92(\mathrm{~d}, 6 \mathrm{H}, J=6.8 \mathrm{~Hz})$ ppm; ${ }^{13} \mathrm{C}$ NMR $\left(\mathrm{CD}_{3} \mathrm{CN}, 125 \mathrm{MHz}, 45^{\circ} \mathrm{C}\right) \delta 158.6,95.5,78.8,49.5,28.9,20.2 \mathrm{ppm}$; IR (thin film) $v$ 3367, 2964, $1594,1404,1317,1190,1089,1021,845,749 \mathrm{~cm}^{-1}$.

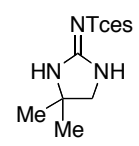

Purified by chromatography on silica gel (gradient elution: $1: 0 \rightarrow 2: 1$ toluene/ $\mathrm{Et}_{2} \mathrm{O}$ ); white solid $(74 \%)$ : $\mathrm{mp} 195{ }^{\circ} \mathrm{C}$; TLC $\mathrm{R}_{f}=0.30(1: 1$ toluene/Et $2 \mathrm{O}) ;{ }^{1} \mathrm{H} \mathrm{NMR}\left(\mathrm{CD}_{3} \mathrm{CN}, 400 \mathrm{MHz}\right) \delta 6.50$ (br s, $\left.1 \mathrm{H}\right), 6.36(\mathrm{br} \mathrm{s}, 1 \mathrm{H}), 4.57$ (s, $\left.2 \mathrm{H}\right), 3.36$ 
(s, 2H), $1.31(\mathrm{~s}, 6 \mathrm{H}) \mathrm{ppm} ;{ }^{13} \mathrm{C}$ NMR $\left(\mathrm{CD}_{3} \mathrm{CN}, 125 \mathrm{MHz}\right) \delta 160.4,95.3,78.5,58.6,55.7,27.5 \mathrm{ppm}$; IR (thin film) $v$ 3393, 3217, 2974, 1598, 1535, 1271, 1159, 1082, 1018, 842, 753, 722, $592 \mathrm{~cm}^{-1}$; HRMS $\left(\mathrm{ES}^{+}\right)$calcd for $\mathrm{C}_{7} \mathrm{H}_{12} \mathrm{Cl}_{3} \mathrm{~N}_{3} \mathrm{O}_{3} \mathrm{~S} 322.9665$ found $345.9549\left(\mathrm{MNa}^{+}\right)$.

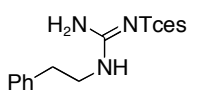

Prepared according to method A. Purified by chromatography on silica gel (gradient elution: $3: 1 \rightarrow 3: 2$ hexanes/EtOAc); white solid (89\%): mp 61-64 ${ }^{\circ} \mathrm{C}$; TLC $\mathrm{R}_{f}=0.27\left(1: 1\right.$ toluene/Et $\left.{ }_{2} \mathrm{O}\right) ;{ }^{1} \mathrm{H} \mathrm{NMR}\left(\mathrm{CDCl}_{3}, 500 \mathrm{MHz}\right.$, $\left.58{ }^{\circ} \mathrm{C}\right) \delta$ 7.39-7.33 (m, 2H), 7.31-7.26 (m, 1H), 7.25-7.21 (m, 2H), $5.89(\mathrm{br} \mathrm{s}, 3 \mathrm{H}), 4.60(\mathrm{~s}, 2 \mathrm{H}), 3.53(\mathrm{q}, 2 \mathrm{H}, J=6.5$ $\mathrm{Hz}), 2.92(\mathrm{t}, 2 \mathrm{H}, J=6.5 \mathrm{~Hz}) \mathrm{ppm} ;{ }^{13} \mathrm{C} \mathrm{NMR}\left(\mathrm{CDCl}_{3}, 125 \mathrm{MHz}, 58{ }^{\circ} \mathrm{C}\right) \delta 157.3,137.7,129.1,128.8,127.2,94.4$, 78.4, 43.3, 35.4 ppm; IR (thin film) v 3461, 3355, 2945, 1550, 1497, 1454, 1406, 1310, 1181, 1132, 1087, 1021, 851 $\mathrm{cm}^{-1}$.

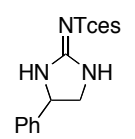

Purified by chromatography on silica gel (gradient elution: $1: 0 \rightarrow 2: 1$ toluene/ $\mathrm{Et}_{2} \mathrm{O}$ ); white solid $\left(57 \%\right.$ ): $\mathrm{mp} 168{ }^{\circ} \mathrm{C}$; TLC $\mathrm{R}_{f}=0.45\left(1: 1\right.$ toluene/Et $\left.{ }_{2} \mathrm{O}\right) ;{ }^{1} \mathrm{H}$ NMR $\left(\mathrm{CD}_{3} \mathrm{CN}, 400 \mathrm{MHz}\right) \delta$ 7.42-7.32 (m, 5H), 6.82 (br s, $\left.1 \mathrm{H}\right), 6.51$ (br s, $1 \mathrm{H}), 5.04(\mathrm{dd}, 1 \mathrm{H}, J=9.2,7.6 \mathrm{~Hz}), 4.63(\mathrm{~s}, 2 \mathrm{H}), 4.03(\mathrm{t}, 1 \mathrm{H}, J=9.6 \mathrm{~Hz}), 3.42(\mathrm{dd}, 1 \mathrm{H}, J=9.6,7.6 \mathrm{~Hz}) \mathrm{ppm} ;{ }^{13} \mathrm{C}$ NMR $\left(\mathrm{CD}_{3} \mathrm{CN}, 125 \mathrm{MHz}, 50{ }^{\circ} \mathrm{C}\right) \delta 162.2,141.9,130.0,129.4,127.3,95.6,79.0,58.7,51.6 \mathrm{ppm}$; IR (thin film) $v$ $3408,3171,1623,1542,1177,1116,1020,840 \mathrm{~cm}^{-1}$; HRMS $\left(\mathrm{ES}^{+}\right)$calcd for $\mathrm{C}_{11} \mathrm{H}_{12} \mathrm{Cl}_{3} \mathrm{~N}_{3} \mathrm{O}_{3} \mathrm{~S} 370.9665$ found $393.9572\left(\mathrm{MNa}^{+}\right)$.

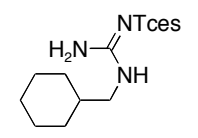

Prepared according to method A. Purified by chromatography on silica gel (gradient elution: $3: 1 \rightarrow 1: 1$ hexanes/EtOAc); white solid (85\%): $\mathrm{mp} 127-128{ }^{\circ} \mathrm{C}$; TLC $\mathrm{R}_{f}=0.30$ (1:1 toluene/Et $\left.\mathrm{E}_{2} \mathrm{O}\right) ;{ }^{1} \mathrm{H}$ NMR $\left(\mathrm{CD}_{3} \mathrm{CN}, 400\right.$ $\left.\mathrm{MHz}, 60{ }^{\circ} \mathrm{C}\right) \delta 6.10(\mathrm{br} \mathrm{s}, 3 \mathrm{H}), 4.59(\mathrm{~s}, 2 \mathrm{H}), 3.04(\mathrm{br} \mathrm{t}, 2 \mathrm{H}, J=5.8 \mathrm{~Hz}), 1.76-1.69(\mathrm{~m}, 4 \mathrm{H}), 1.69-1.63$ (m, 1H), 1.59$1.48(\mathrm{~m}, 1 \mathrm{H}), 1.33-1.13(\mathrm{~m}, 3 \mathrm{H}), 1.03-0.92(\mathrm{~m}, 2 \mathrm{H}) \mathrm{ppm} ;{ }^{13} \mathrm{C} \mathrm{NMR}\left(\mathrm{CD}_{3} \mathrm{CN}, 125 \mathrm{MHz}, 65{ }^{\circ} \mathrm{C}\right) \delta 159.0,95.8,79.1$, 48.7, 38.6, 31.6, 27.4, $26.7 \mathrm{ppm}$; IR (thin film) v 3465, 3376, 3267, 2928, 1643, 1597, 1326, 1191, 1140, $833 \mathrm{~cm}^{-1}$.

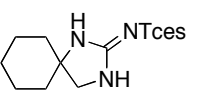

Purified by chromatography on silica gel (gradient elution: $1: 0 \rightarrow 2: 1$ toluene/ $\mathrm{Et}_{2} \mathrm{O}$ ); white solid (80\%): $\mathrm{mp} 212-214$ ${ }^{\circ} \mathrm{C}$; TLC $\mathrm{R}_{f}=0.44(1: 1$ toluene/Et $2 \mathrm{O}) ;{ }^{1} \mathrm{H}$ NMR $\left(\mathrm{CD}_{3} \mathrm{CN}, 400 \mathrm{MHz}\right) \delta 6.53$ (br s, $\left.1 \mathrm{H}\right), 6.43$ (br s, $\left.1 \mathrm{H}\right), 4.57$ (s, 2H), $3.40(\mathrm{~s}, 2 \mathrm{H}), 1.70-1.54(\mathrm{~m}, 6 \mathrm{H}), 1.49-1.25(\mathrm{~m}, 4 \mathrm{H}) \mathrm{ppm} ;{ }^{13} \mathrm{C} \mathrm{NMR}\left(\mathrm{CDCl}_{3}, 125 \mathrm{MHz}, 50{ }^{\circ} \mathrm{C}\right) \delta 159.6,94.5,78.3$, 61.2, 52.9, 37.1, 24.7, 22.8 ppm; IR (thin film) v 3400, 3225, 2931, 1609, 1537, 1327, 1169, 1027, $848 \mathrm{~cm}^{-1}$; HRMS $\left(\mathrm{ES}^{+}\right)$calcd for $\mathrm{C}_{10} \mathrm{H}_{10} \mathrm{Cl}_{3} \mathrm{~N}_{3} \mathrm{O}_{3} \mathrm{~S} 362.9978$ found $385.9863\left(\mathrm{MNa}^{+}\right)$.

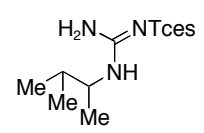

Prepared according to method B. Purified by chromatography on silica gel (gradient elution: 6:1 $\rightarrow 3: 1$ hexanes/EtOAc); white solid (92\%): $\mathrm{mp} 83-85{ }^{\circ} \mathrm{C}$; $\mathrm{TLC} \mathrm{R}_{f}=0.26(1: 1$ toluene/Et $2 \mathrm{O}) ;{ }^{1} \mathrm{H}$ NMR $\left(\mathrm{CD}_{3} \mathrm{CN}, 400 \mathrm{MHz}\right.$, $\left.65^{\circ} \mathrm{C}\right) \delta 6.10(\mathrm{br} \mathrm{s}, 3 \mathrm{H}), 4.59(\mathrm{~s}, 2 \mathrm{H}), 3.62(\mathrm{br} \mathrm{s}, 1 \mathrm{H}), 1.81-1.69(\mathrm{~m} \mathrm{1H}), 1.10(\mathrm{~d}, 3 \mathrm{H}, J=6.4 \mathrm{~Hz}), 0.92(\mathrm{~d}, 3 \mathrm{H}, J=$ $6.8 \mathrm{~Hz}), 0.91(\mathrm{~d}, 3 \mathrm{H}, J=6.8 \mathrm{~Hz}) \mathrm{ppm} ;{ }^{13} \mathrm{C} \mathrm{NMR}\left(\mathrm{CD}_{3} \mathrm{CN}, 125 \mathrm{MHz}, 60{ }^{\circ} \mathrm{C}\right) \delta 158.3,95.7,79.0,54.0,34.0,18.9$, 17.81, 17.76 ppm; IR (thin film) v 3462, 3356, 2967, 1630, 1548, 1406, 1309, 1184, 1089, 1022, $852 \mathrm{~cm}^{-1}$. 


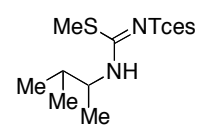

Purified by chromatography on silica gel (gradient elution: 9:1 $\rightarrow 5: 1$ hexanes/EtOAc); clear oil (96\%): TLC $\mathrm{R}_{f}=$ 0.55 (3:1 hexanes/EtOAc); ${ }^{1} \mathrm{H}$ NMR $\left(\mathrm{CDCl}_{3}, 400 \mathrm{MHz}\right) \delta 7.84$ (br d, $\left.1 \mathrm{H}, J=9.2 \mathrm{~Hz}\right), 4.63(\mathrm{~s}, 2 \mathrm{H}), 3.60-3.51$ (m, $1 \mathrm{H}), 2.44(\mathrm{~s}, 3 \mathrm{H}), 1.84-1.76(\mathrm{~m}, 1 \mathrm{H}), 1.19(\mathrm{~d}, 3 \mathrm{H}, J=6.4 \mathrm{~Hz}), 0.95(\mathrm{~d}, 3 \mathrm{H}, J=6.8 \mathrm{~Hz}), 0.93(\mathrm{~d}, 3 \mathrm{H}, J=6.4 \mathrm{~Hz})$ ppm; IR (thin film) v 3296, 2966, 1572, 1429, 1344, 1140, 1088, 1021, 870, $751 \mathrm{~cm}^{-1}$.

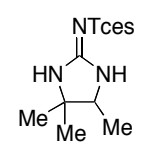

Purified by chromatography on silica gel (gradient elution: $1: 0 \rightarrow 2: 1$ toluene/Et ${ }_{2} \mathrm{O}$ ); white solid (54\%): $\mathrm{mp}$ 201-202 ${ }^{\circ} \mathrm{C}$; $\mathrm{TLC} \mathrm{R}_{f}=0.39(1: 1$ toluene/Et $2 \mathrm{O}) ;{ }^{1} \mathrm{H} \mathrm{NMR}\left(\mathrm{CDCl}_{3}, 500 \mathrm{MHz}, 50{ }^{\circ} \mathrm{C}\right) \delta 6.69$ (br s, $\left.1 \mathrm{H}\right), 6.60$ (br s, $\left.1 \mathrm{H}\right), 4.57$ (s, 2H), $3.67(\mathrm{q}, 1 \mathrm{H}, J=6.5 \mathrm{~Hz}), 1.35(\mathrm{~s}, 3 \mathrm{H}), 1.23-1.19(\mathrm{~m}, 6 \mathrm{H}) \mathrm{ppm} ;{ }^{13} \mathrm{C} \mathrm{NMR}\left(\mathrm{CDCl}_{3}, 125 \mathrm{MHz}, 50{ }^{\circ} \mathrm{C}\right) \delta 159.2$, 94.4, 78.3, 60.7, 60.0, 27.8, 22.1, 15.0 ppm; IR (thin film) v 3383, 3234, 1637, 1513, 1318, 1171, 1088, 1024, 862 $\mathrm{cm}^{-1}$; HRMS $\left(\mathrm{ES}^{+}\right)$calcd for $\mathrm{C}_{8} \mathrm{H}_{14} \mathrm{Cl}_{3} \mathrm{~N}_{3} \mathrm{O}_{3} \mathrm{~S} 336.9821$ found $359.9709\left(\mathrm{MNa}^{+}\right)$.

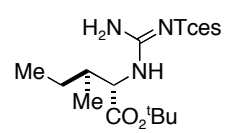

Prepared according to method B. Purified by chromatography on silica gel (gradient elution: 6:1 $\rightarrow 3: 1$ hexanes/EtOAc); white solid (89\%): $\mathrm{mp} 148-150{ }^{\circ} \mathrm{C}$; $\mathrm{TLC} \mathrm{R}_{f}=0.48\left(1: 1\right.$ toluene/ $\left./ \mathrm{Et}_{2} \mathrm{O}\right) ;{ }^{1} \mathrm{H} \mathrm{NMR}\left(\mathrm{CD}_{3} \mathrm{CN}, 500\right.$ $\left.\mathrm{MHz}, 65^{\circ} \mathrm{C}\right) \delta 6.25(\mathrm{br} \mathrm{s}, 2 \mathrm{H}), 6.08(\mathrm{br} \mathrm{s}, 1 \mathrm{H}), 4.58(\mathrm{~s}, 2 \mathrm{H}), 4.23(\mathrm{br} \mathrm{s}, 1 \mathrm{H}), 1.91-1.83(\mathrm{~m}, 1 \mathrm{H}), 1.54-1.45(\mathrm{~m}, 1 \mathrm{H})$, $1.47(\mathrm{~s}, 9 \mathrm{H}), 1.30-1.20(\mathrm{~m}, 1 \mathrm{H}), 0.97-0.92(\mathrm{~m}, 6 \mathrm{H}) \mathrm{ppm} ;{ }^{13} \mathrm{C} \mathrm{NMR}\left(\mathrm{CD}_{3} \mathrm{CN}, 125 \mathrm{MHz}, 65{ }^{\circ} \mathrm{C}\right) \delta 171.2,158.6,95.7$, $83.3,79.2,60.2,38.8,28.6,26.5,16.1,12.1$ ppm; IR (thin film) v 3473, 3372, 2980, 2361, 1699, 1617, 1580, 1534, $1339,1186,1088,1031,853 \mathrm{~cm}^{-1}$.

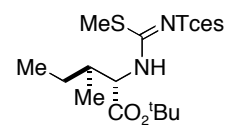

Purified by chromatography on silica gel (gradient elution: 9:1 $\rightarrow 5: 1$ hexanes/EtOAc); clear oil (97\%): $\mathrm{TLC} \mathrm{R}_{f}=$ $0.52\left(3: 1\right.$ hexanes/EtOAc); ${ }^{1} \mathrm{H} \mathrm{NMR}\left(\mathrm{CDCl}_{3}, 400 \mathrm{MHz}\right) \delta 8.42(\mathrm{br} \mathrm{d}, 1 \mathrm{H}, J=8.8 \mathrm{~Hz}), 4.65(\mathrm{~s}, 2 \mathrm{H}), 4.10(\mathrm{dd}, 1 \mathrm{H}, J=$ 8.6, $4.6 \mathrm{~Hz}), 2.45(\mathrm{~s}, 3 \mathrm{H}), 1.96-1.92(\mathrm{~m}, 1 \mathrm{H}), 1.54-1.47(\mathrm{~m}, 1 \mathrm{H}), 1.47(\mathrm{~s}, 9 \mathrm{H}), 1.32-1.24(\mathrm{~m}, 1 \mathrm{H}), 0.96-0.93(\mathrm{~m}, 6 \mathrm{H})$ ppm; IR (thin film) v 3296, 2970, 1735, 1571, 1431, 1343, 1158, 1088, 1021, $876 \mathrm{~cm}^{-1}$.

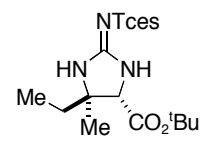

Purified by chromatography on silica gel (gradient elution: 1:0 $\rightarrow 4: 1$ toluene/ $\mathrm{Et}_{2} \mathrm{O}$ ); white solid (91\%): $\mathrm{mp} 139-140$ ${ }^{\circ} \mathrm{C}$; TLC $\mathrm{T}_{f}=0.58(1: 1$ toluene/Et $2 \mathrm{O})$; ${ }^{1} \mathrm{H}$ NMR $\left(\mathrm{CDCl}_{3}, 400 \mathrm{MHz}\right) \delta 6.60$ (br s, $\left.1 \mathrm{H}\right), 6.49$ (br s, $\left.1 \mathrm{H}\right), 4.63(\mathrm{~d}, 1 \mathrm{H}, J=$ $11.2 \mathrm{~Hz}), 4.57(\mathrm{~d}, 1 \mathrm{H}, J=11.2 \mathrm{~Hz}), 4.10(\mathrm{~s}, 1 \mathrm{H}), 1.76(\mathrm{q}, 2 \mathrm{H}, J=7.6 \mathrm{~Hz}), 1.48(\mathrm{~s}, 9 \mathrm{H}), 1.29(\mathrm{~s}, 3 \mathrm{H}), 0.98(\mathrm{t}, 3 \mathrm{H}, J=$ $7.4 \mathrm{~Hz}) \mathrm{ppm} ;{ }^{13} \mathrm{C} \mathrm{NMR}\left(\mathrm{CDCl}_{3}, 500 \mathrm{MHz}\right) \delta 167.6,159.1,94.1,83.5,78.2,64.2,63.5,34.3,28.0,22.1,8.0 \mathrm{ppm}$; IR (thin film) $v 3365,3168,2978,1744,1623,1521,1315,1231,1158,1019,914,845 \mathrm{~cm}^{-1}$; HRMS $\left(\mathrm{ES}^{+}\right.$) calcd for $\mathrm{C}_{13} \mathrm{H}_{22} \mathrm{Cl}_{3} \mathrm{~N}_{3} \mathrm{O}_{5} \mathrm{~S} 437.0346$ found $460.0253\left(\mathrm{MNa}^{+}\right)$. 


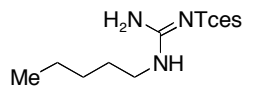

Prepared according to method A. Purified by chromatography on silica gel (gradient elution: $3: 1 \rightarrow 2: 1$

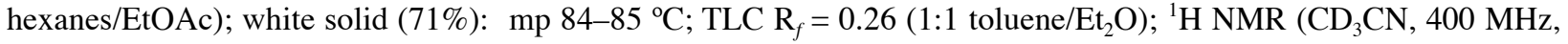
$\left.70{ }^{\circ} \mathrm{C}\right) \delta 6.13$ (br s, 3H), 4.59 (s, 2H), 3.18 (dt, 2H, $\left.J=6.8,5.6 \mathrm{~Hz}\right), 1.58-1.50$ (m, 2H), 1.38-1.30 (m, 4H), 0.91 (t, $3 \mathrm{H}, J=6.8 \mathrm{~Hz}) \mathrm{ppm} ;{ }^{13} \mathrm{C} \mathrm{NMR}\left(\mathrm{CD}_{3} \mathrm{CN}, 125 \mathrm{MHz}, 60{ }^{\circ} \mathrm{C}\right) \delta 158.8,95.8,79.0,42.5,29.7,29.6,23.1,14.4 \mathrm{ppm} ; \mathrm{IR}$ (thin film) $v$ 3461, 3355, 2934, 2871, 1554, 1406, 1307, 1186, 1021, $861 \mathrm{~cm}^{-1}$.

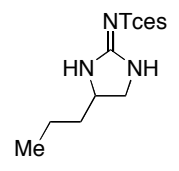

Purified by chromatography on silica gel (gradient elution: 1:0 $\rightarrow 2: 1$ toluene/Et $2 \mathrm{O}$ ); white solid (30\%): mp 98-99 ${ }^{\circ} \mathrm{C}$; TLC $\mathrm{R}_{f}=0.47(1: 1$ toluene/Et $2 \mathrm{O}) ;{ }^{1} \mathrm{H} \mathrm{NMR}\left(\mathrm{CDCl}_{3}, 500 \mathrm{MHz}\right) \delta 6.60($ br s, $1 \mathrm{H}), 5.98(\mathrm{br} \mathrm{s}, 1 \mathrm{H}), 4.58(\mathrm{~d}, 1 \mathrm{H}, J$ $=11.0 \mathrm{~Hz}), 4.56(\mathrm{~d}, 1 \mathrm{H}, J=11.0 \mathrm{~Hz}), 4.02-3.96(\mathrm{~m}, 1 \mathrm{H}), 3.78(\mathrm{t}, 1 \mathrm{H}, J=9.5 \mathrm{~Hz}), 3.33(\mathrm{dd}, 1 \mathrm{H}, J=9.3,7.3 \mathrm{~Hz})$, $1.68-1.60(\mathrm{~m}, 1 \mathrm{H}), 1.58-1.50(\mathrm{~m}, 1 \mathrm{H}), 1.39-1.28(\mathrm{~m}, 2 \mathrm{H}), 0.94(\mathrm{t}, 3 \mathrm{H}, J=7.5 \mathrm{~Hz}) \mathrm{ppm} ;{ }^{13} \mathrm{C} \mathrm{NMR}\left(\mathrm{CDCl}_{3}, 125 \mathrm{MHz}\right)$ $\delta$ 160.6, 94.2, 78.1, 54.9, 47.8, 37.3, 18.5, 13.9 ppm; IR (thin film) v 3393, 3243, 2962, 1613, 1533, 1327, 1265, $1175,1087,1026,915,849 \mathrm{~cm}^{-1}$; HRMS $\left(\mathrm{ES}^{+}\right)$calcd for $\mathrm{C}_{8} \mathrm{H}_{14} \mathrm{Cl}_{3} \mathrm{~N}_{3} \mathrm{O}_{3} \mathrm{~S} 336.9821$ found $359.9712\left(\mathrm{MNa}^{+}\right)$.

\section{Experimental Procedure for Tces Guanidine Deprotection}

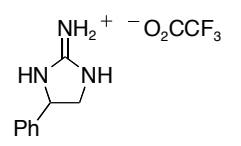

To a suspension of powdered $\mathrm{Zn}$ (44 mg, $0.67 \mathrm{mmol}, 5.0$ equiv) in $1.3 \mathrm{~mL}$ of a 1:1 MeOH/AcOH solution was added 2-trichloroethoxysulfonylamino-4-phenylimidazoline $(50 \mathrm{mg}, 0.13 \mathrm{mmol})$. The mixture was stirred vigorously at $40{ }^{\circ} \mathrm{C}$ for $16 \mathrm{~h}$. The reaction was then cooled to $23^{\circ} \mathrm{C}$ and diluted with $1 \mathrm{~mL}$ of $\mathrm{MeOH}$, and the contents filtered through a small pad of Celite. The flask and filter cake were rinsed with an additional $5 \mathrm{~mL}$ of $\mathrm{MeOH}$. The combined filtrates were concentrated under reduced pressure to give a solid white residue. Purification of the product was performed by reverse phase HPLC on a Varian ProStar 210 Instrument with an Alltima C18 10 $\mu \mathrm{m}$ column $(22 \times 250 \mathrm{~mm})$. A product retention time of 8.46 min was recorded using a 19:1 $\rightarrow 1: 9 \mathrm{H}_{2} \mathrm{O} / \mathrm{MeCN}$ buffered with $0.1 \% \mathrm{CF}_{3} \mathrm{CO}_{2} \mathrm{H}$ gradient elution over $14.60 \mathrm{~min}$ (white solid, $\left.34 \mathrm{mg}, 91 \%\right)$ : ${ }^{1} \mathrm{H}$ NMR $\left(\mathrm{CD}_{3} \mathrm{OD}, 400\right.$ $\mathrm{MHz}) \delta 7.43-7.33(\mathrm{~m}, 5 \mathrm{H}), 5.11(\mathrm{dd}, 1 \mathrm{H}, J=9.6,7.2 \mathrm{~Hz}), 4.09(\mathrm{t}, 1 \mathrm{H}, J=9.8 \mathrm{~Hz}), 3.47(\mathrm{dd}, 1 \mathrm{H}, J=9.6,7.2 \mathrm{~Hz})$ ppm; ${ }^{19} \mathrm{~F}$ NMR ( $\mathrm{CD}_{3} \mathrm{OD}, 376 \mathrm{MHz}$, referenced to $\alpha, \alpha, \alpha$-trifluorotoluene) $\delta-77.4 \mathrm{ppm}$; IR (KBr pellet) $v 3367$, 1680, 1584, 1493, 1458, 1376, 1122, $881 \mathrm{~cm}^{-1}$; HRMS $\left(\mathrm{ES}^{+}\right)$calcd for $\mathrm{C}_{9} \mathrm{H}_{11} \mathrm{~N}_{3} 161.0953$ found $162.1028\left(\mathrm{MH}^{+}\right)$.

(1) Guthikonda, K.; Du Bois, J. J. Am. Chem. Soc. 2002, 124, 13672-13673.

(2) (a) Gautun, H. S. H.; Bergan, T.; Carlsen, P. H. J. Acta Chem. Scand., 1999, 53, 446-452. (b) Lohaus, G. Chem. Ber. 1972, 105, 2791-2799. 
$\mathrm{Cl}_{3} \mathrm{C}_{\mathrm{H}}^{\mathrm{O}} \mathrm{NH}_{2}$

${ }^{1} \mathrm{H}$ NMR

$\left(\mathrm{CD}_{3} \mathrm{CN}, 400 \mathrm{MHz}\right)$

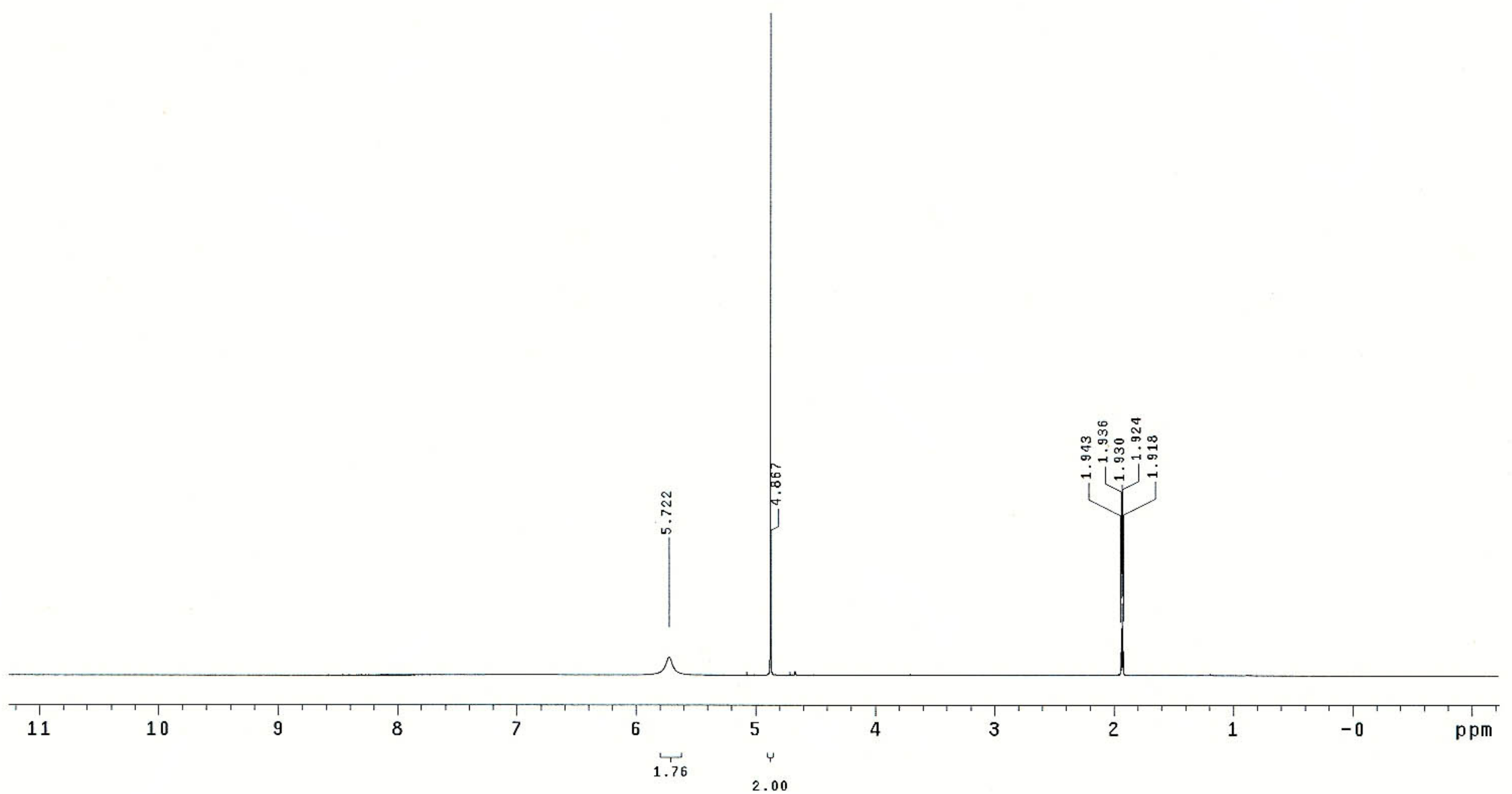




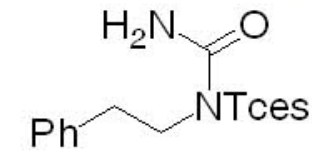

${ }^{1} \mathrm{H}$ NMR

$\left(\mathrm{CDCl}_{3}, 400 \mathrm{MHz}\right)$

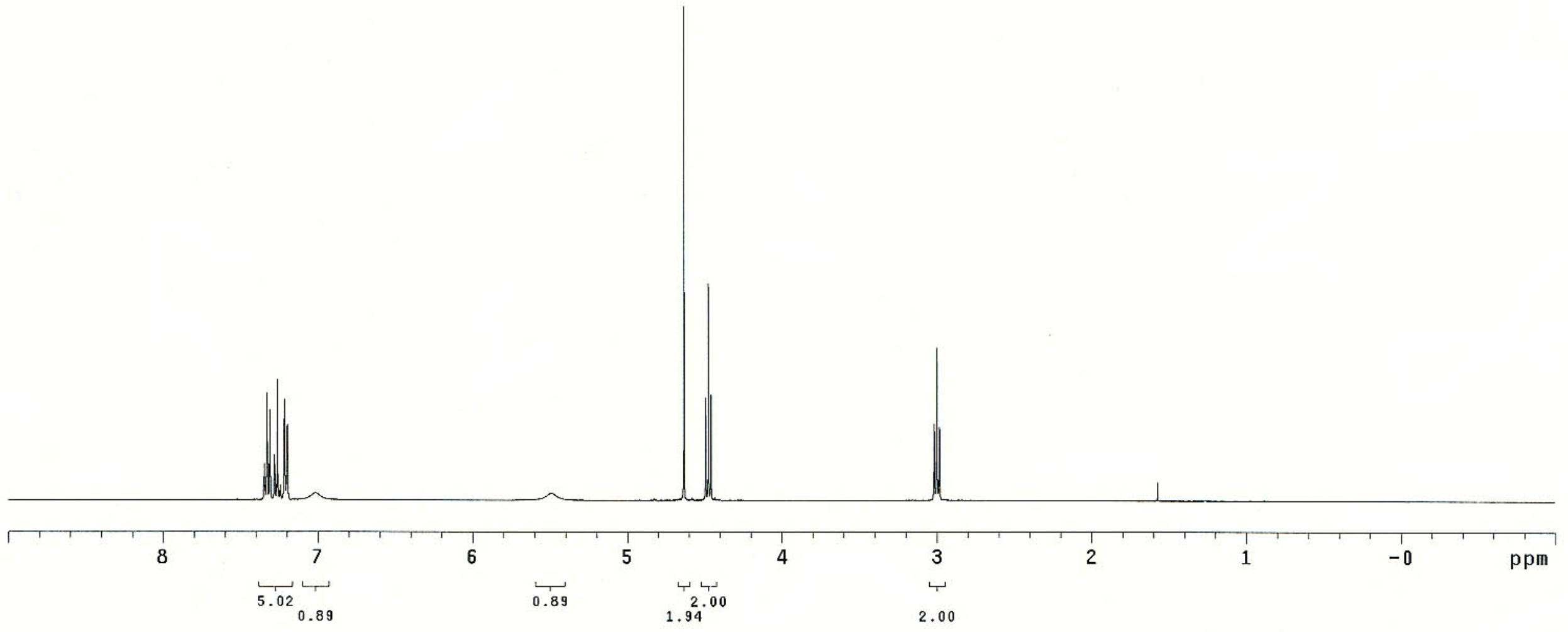



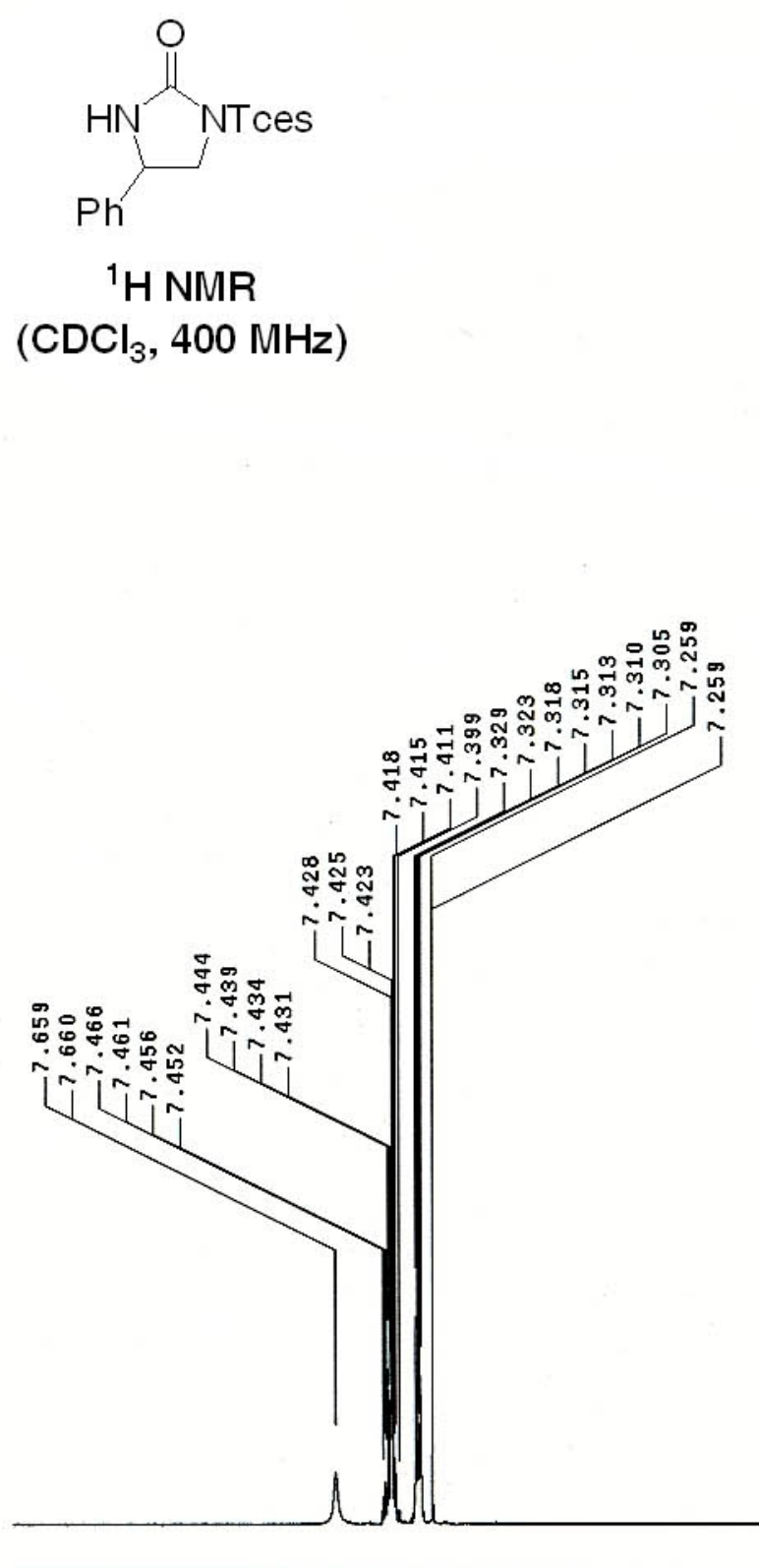

$$
\begin{gathered}
4.89 \\
0.78 \\
2.88
\end{gathered}
$$
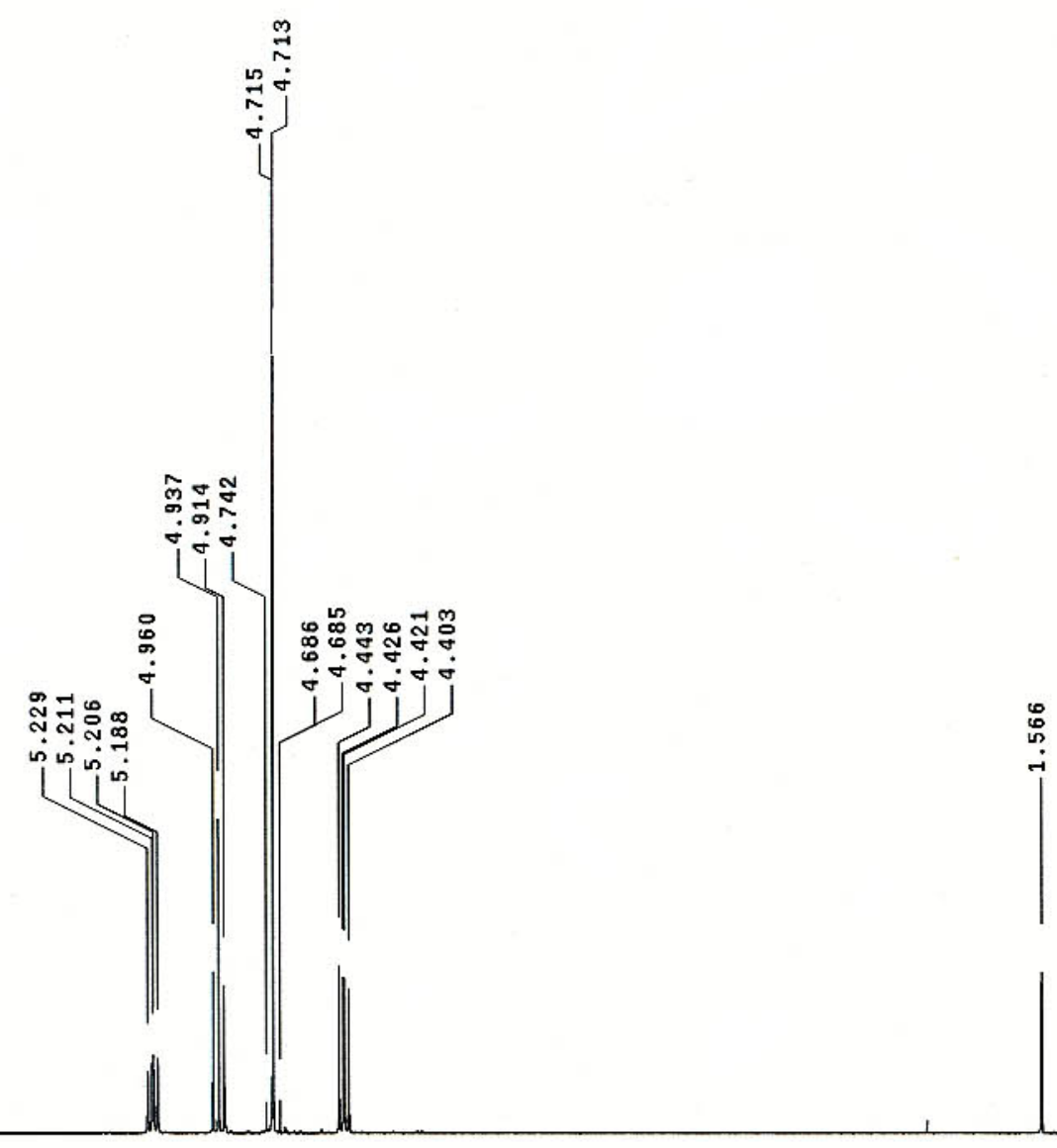

$0.99^{1.03} 1.951 .00$ 


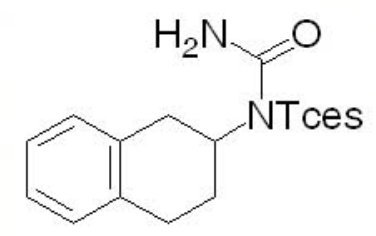

${ }^{1} \mathrm{H}$ NMR

$\left(\mathrm{CDCl}_{3}, 400 \mathrm{MHz}\right)$

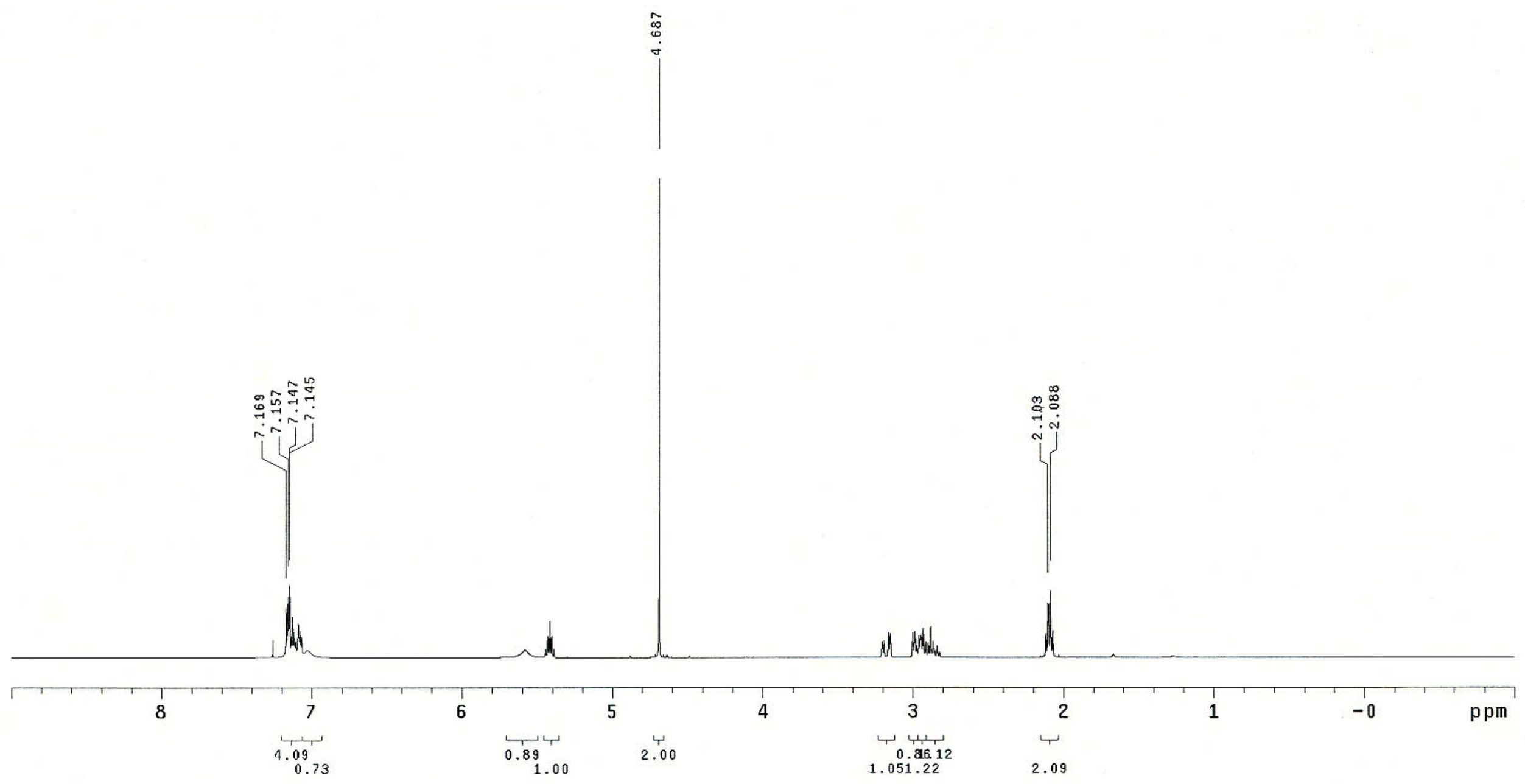



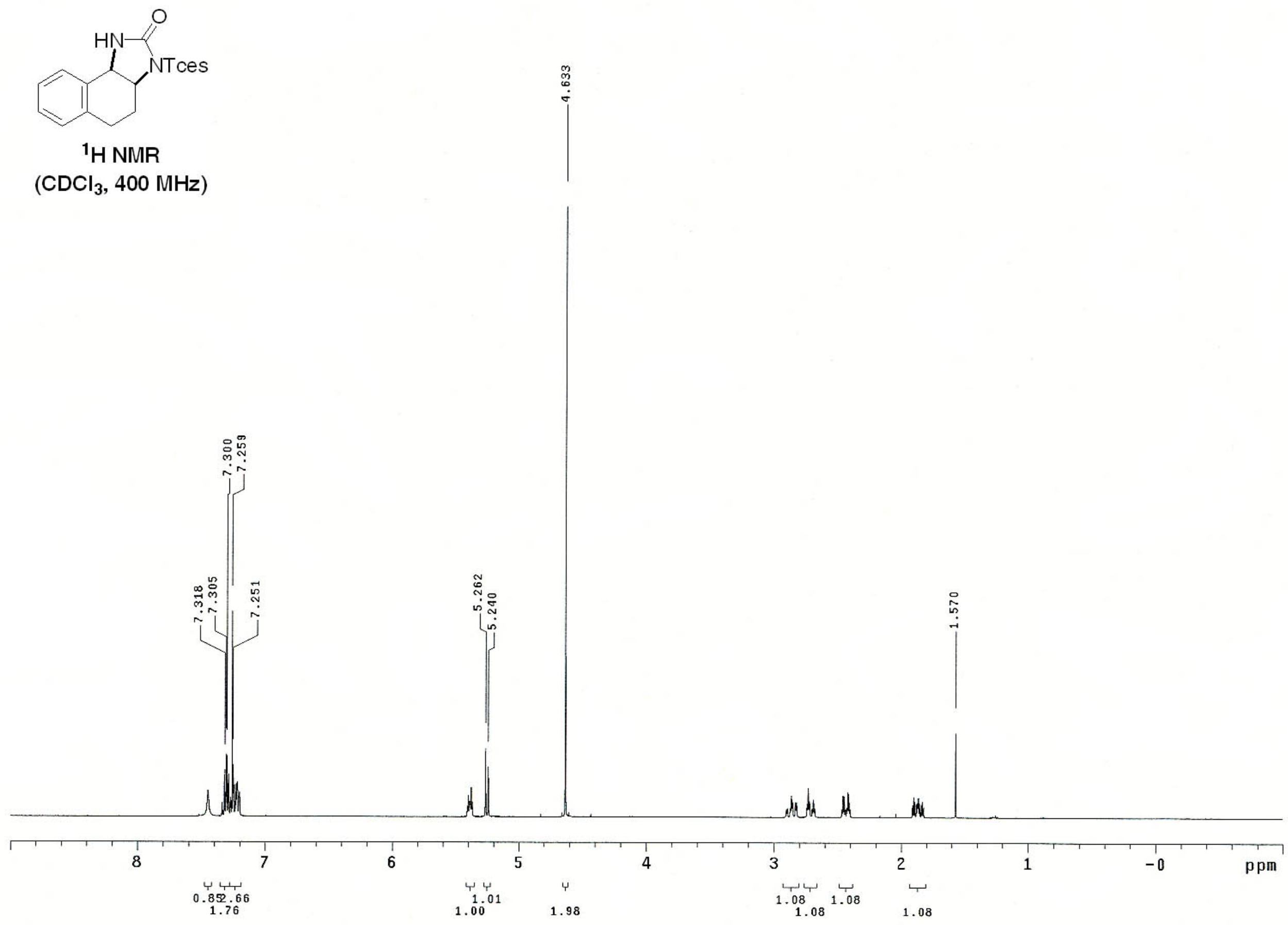


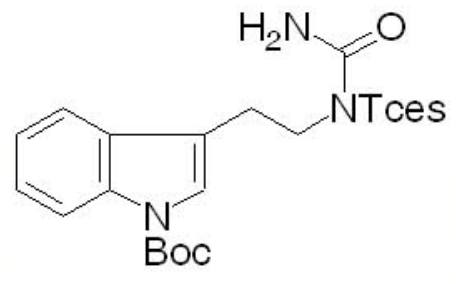

${ }^{1} \mathrm{H}$ NMR

$\left(\mathrm{CDCl}_{3}, 400 \mathrm{MHz}\right)$

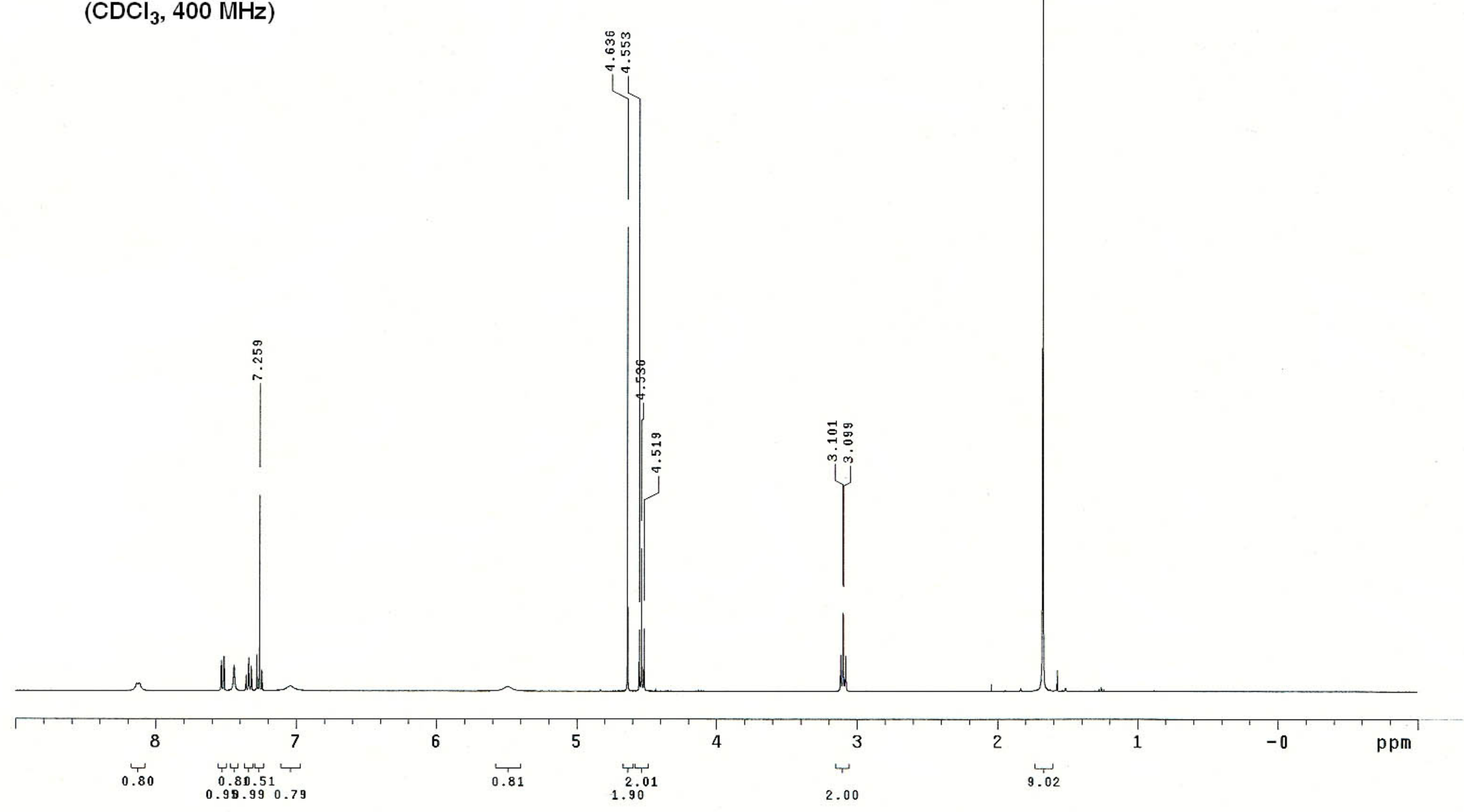




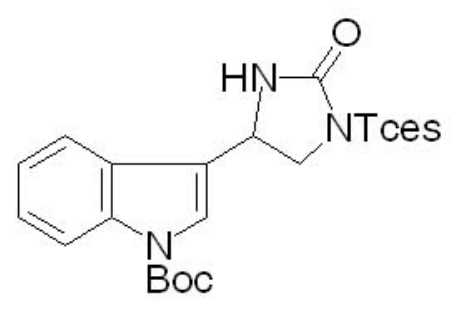

${ }^{1} \mathrm{H}$ NMR

$\left(\mathrm{CDCl}_{3}, 400 \mathrm{MHz}\right)$

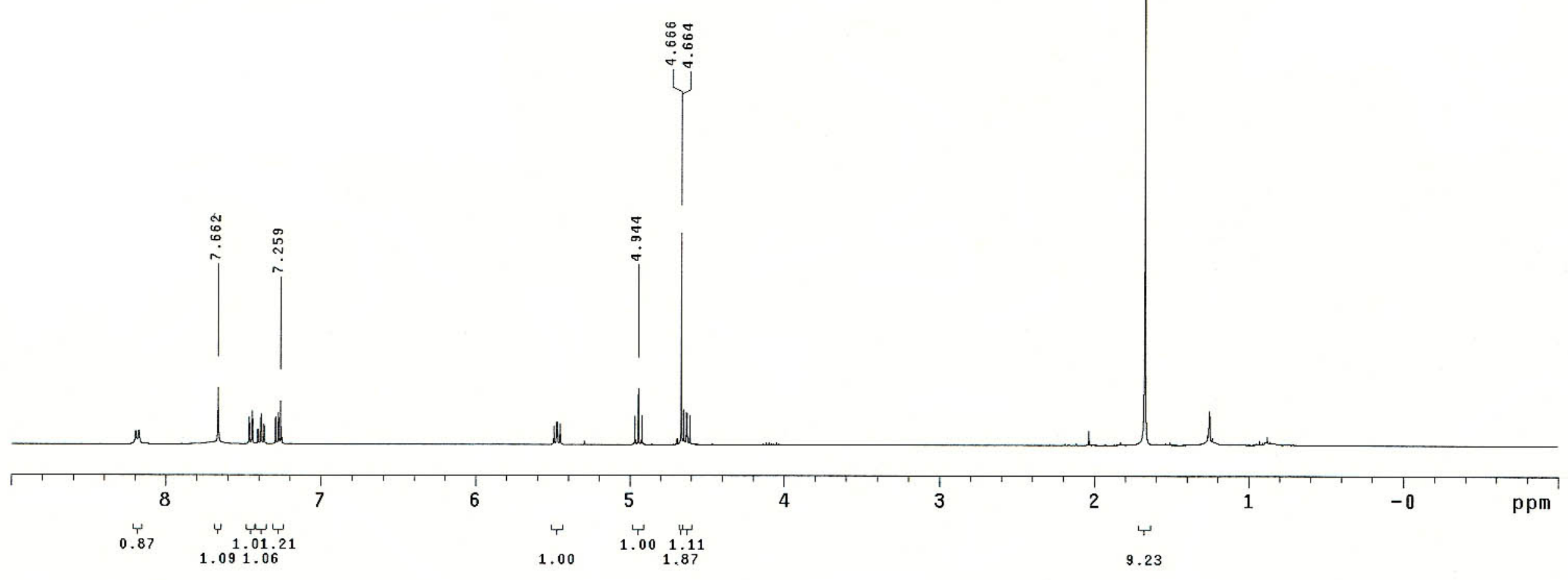


$\mathrm{Me} \overbrace{\mathrm{Me}}^{\mathrm{NT}}$

${ }^{1} \mathrm{H}$ NMR

$\left(\mathrm{CDCl}_{3}, 400 \mathrm{MHz}\right)$

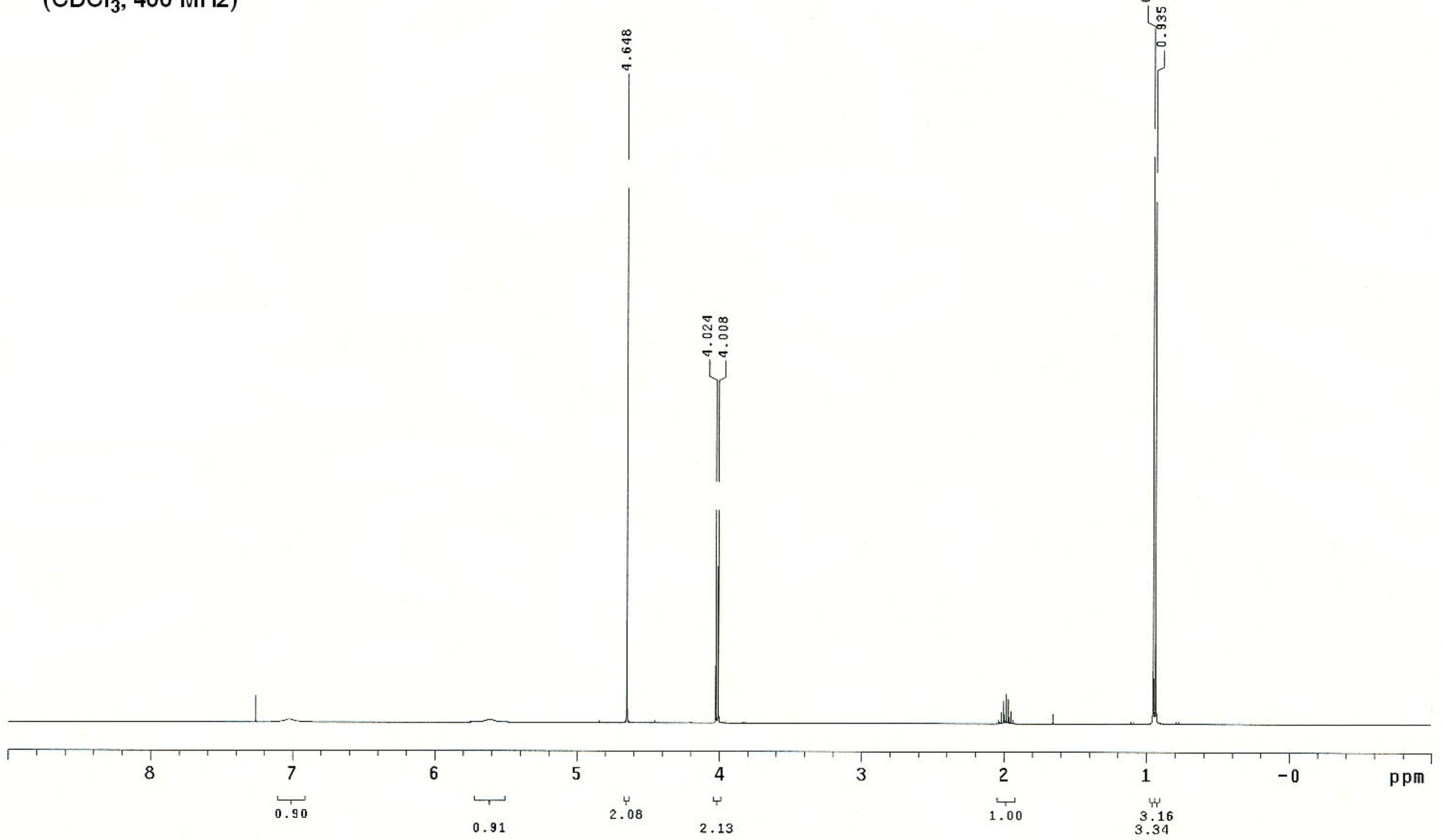




$$
\begin{gathered}
{ }_{\left(\mathrm{CDCl}_{3}, 400 \mathrm{MHz}\right)} \\
{ }^{1} \mathrm{H} \mathrm{NMR}
\end{gathered}
$$

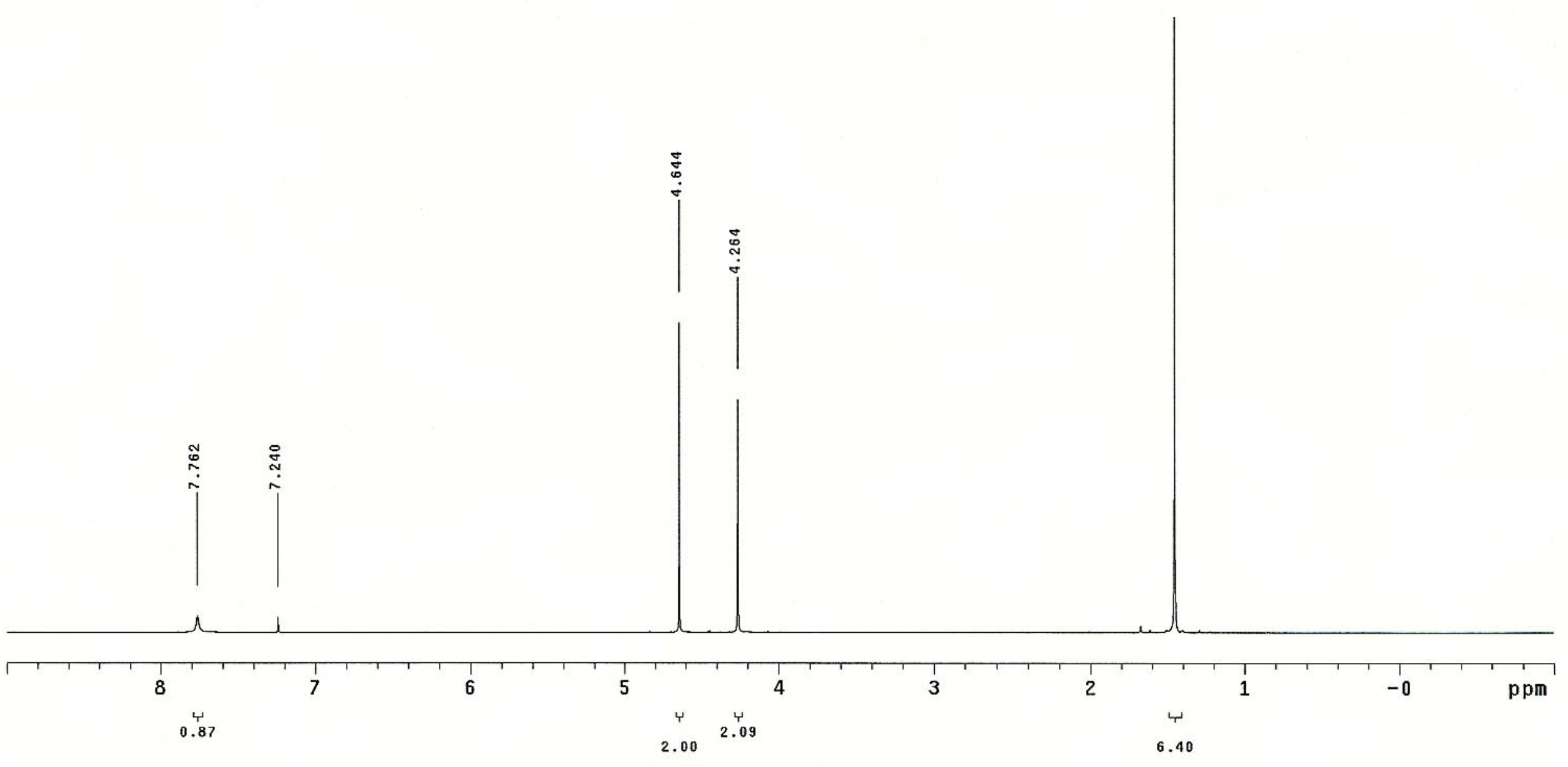


$\mathrm{Me} \mathrm{Me}_{\mathrm{NT} \text { ces }}$

${ }^{1} \mathrm{H}$ NMR

$\left(\mathrm{CDCl}_{3}, 400 \mathrm{MHz}\right)$

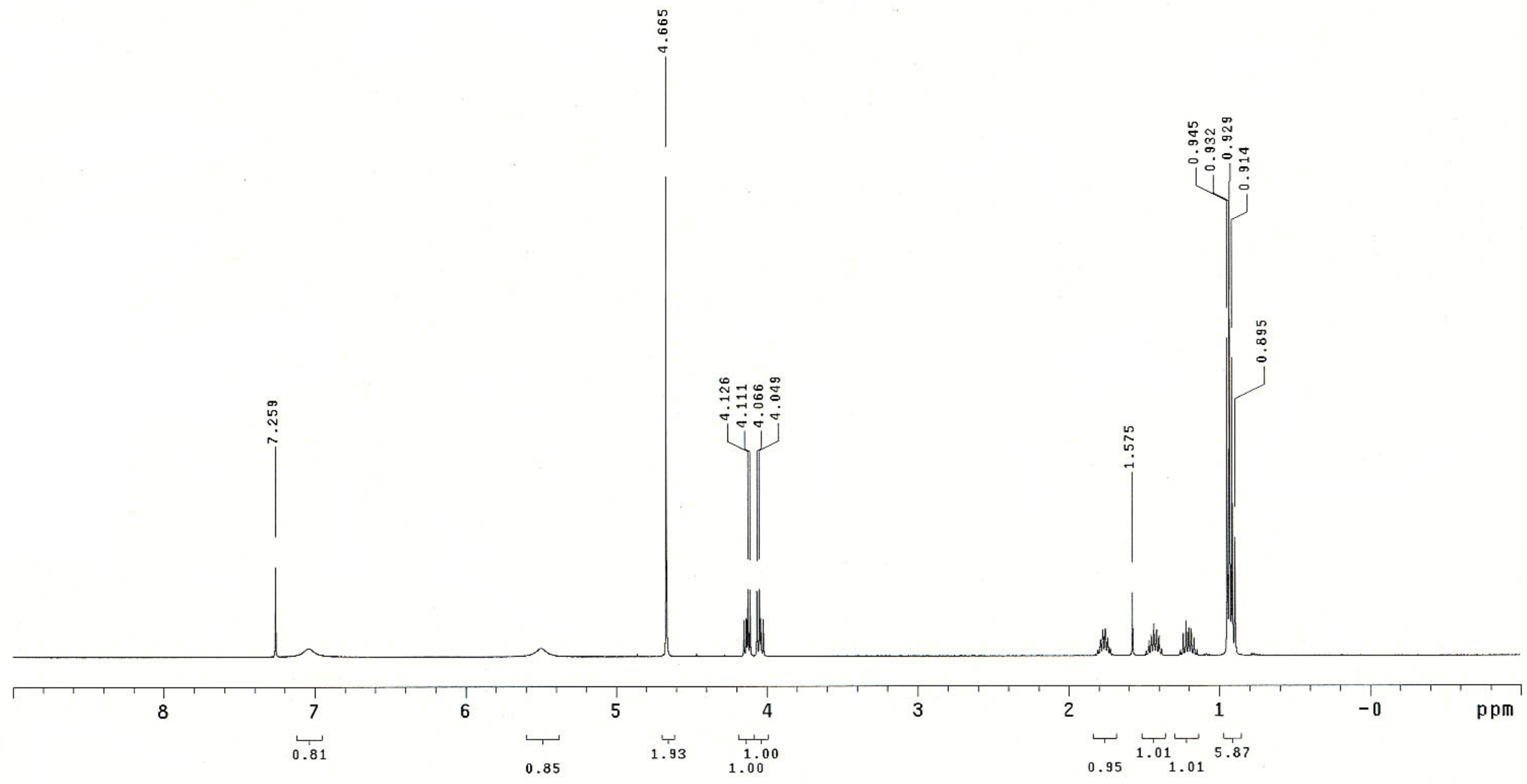


S21<smiles>CCC1(C)C[N+]([Se])C(=O)N1</smiles>

${ }^{1} \mathrm{H}$ NMR

$\left(\mathrm{CDCl}_{3}, 400 \mathrm{MHz}\right)$

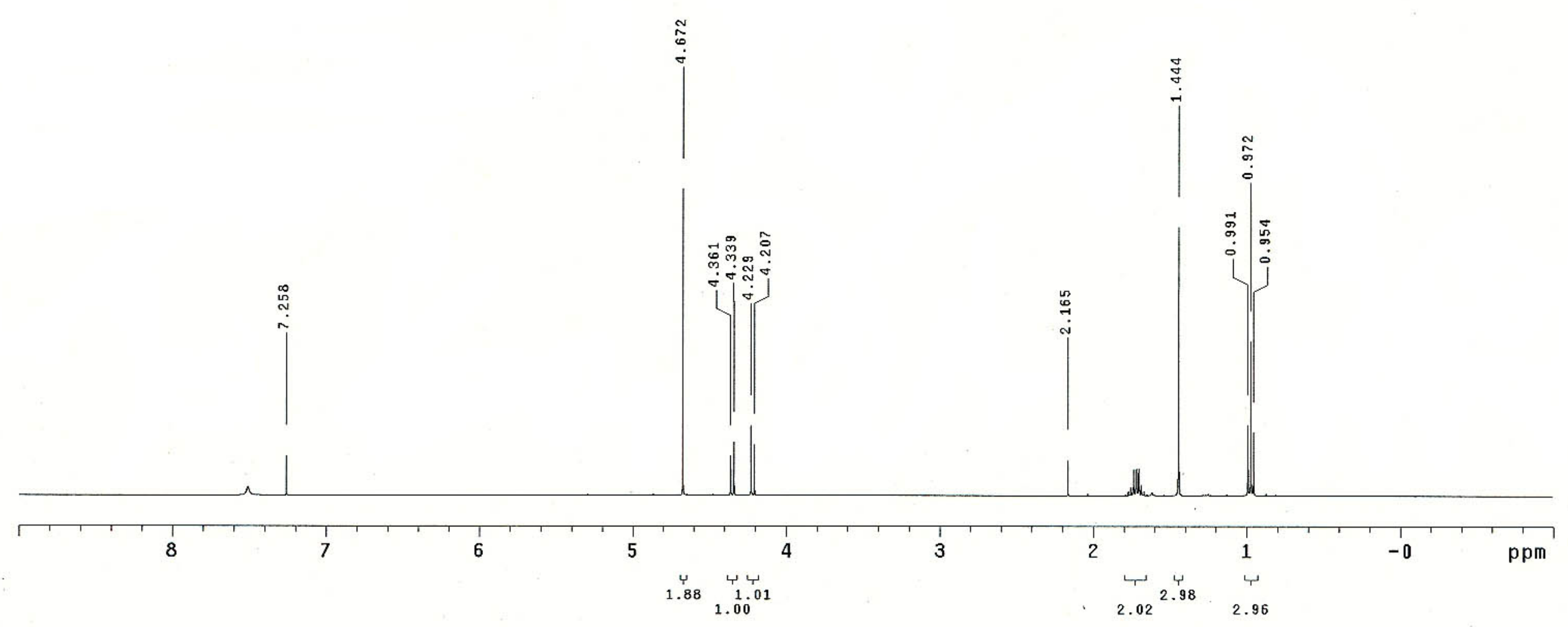




$$
{ }_{\left(\mathrm{CDCl}_{3}, 400 \mathrm{MHz}\right)}^{{ }^{1} \mathrm{H} \text { NMR }}
$$

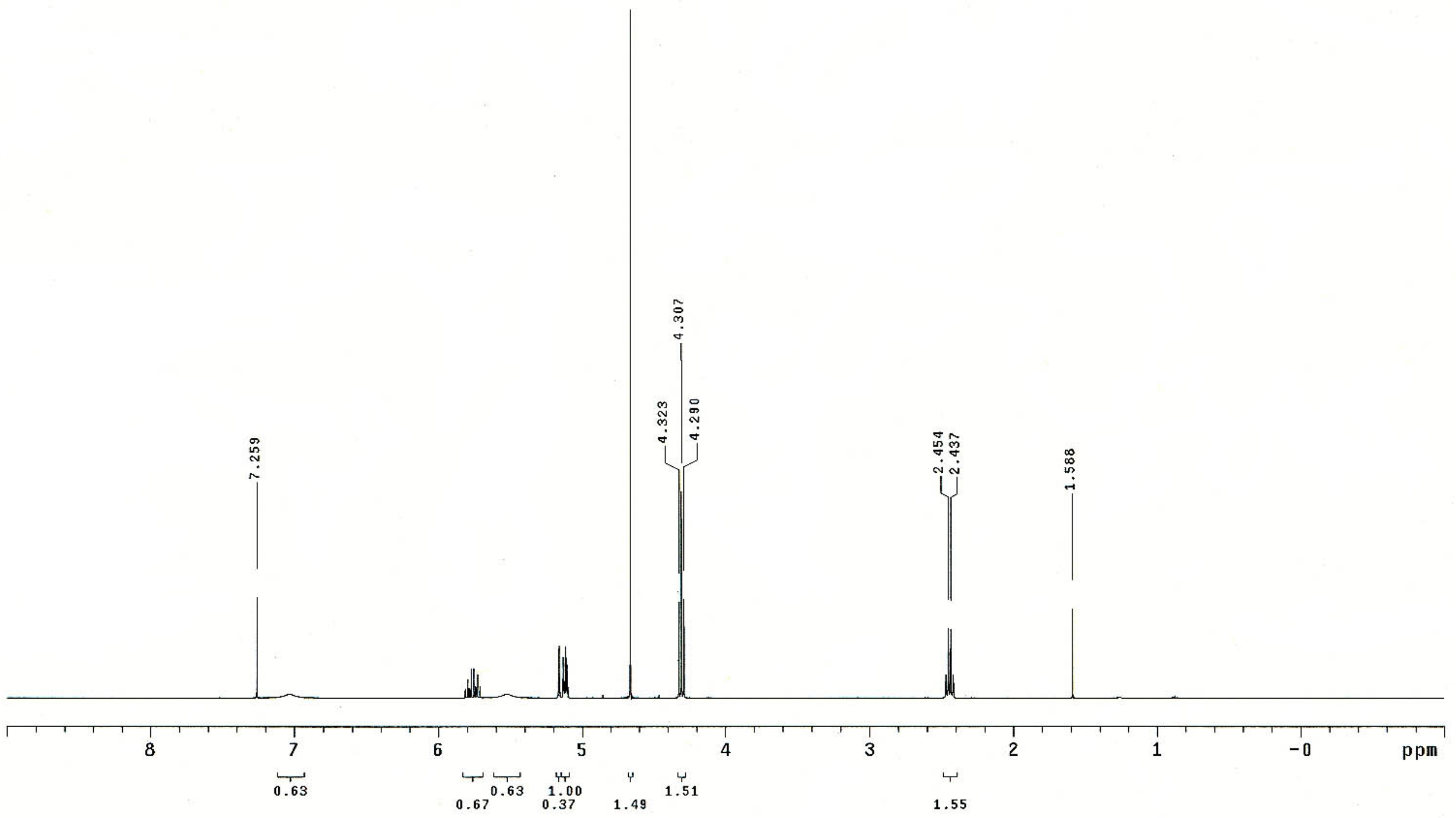




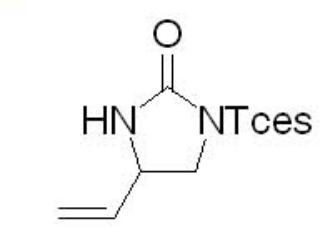

${ }^{1} \mathrm{H}$ NMR

$\left(\mathrm{CDCl}_{3}, 400 \mathrm{MHz}\right)$

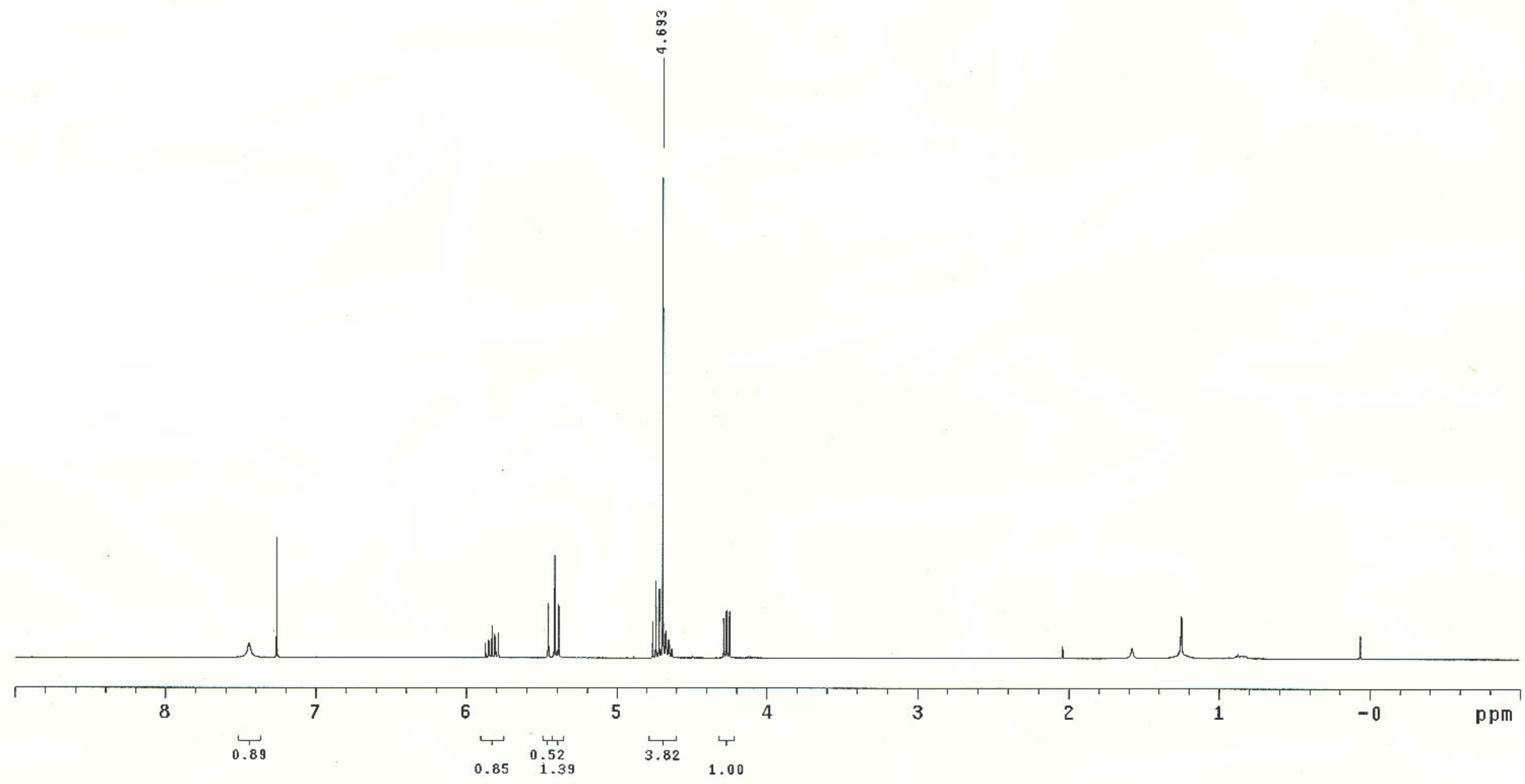




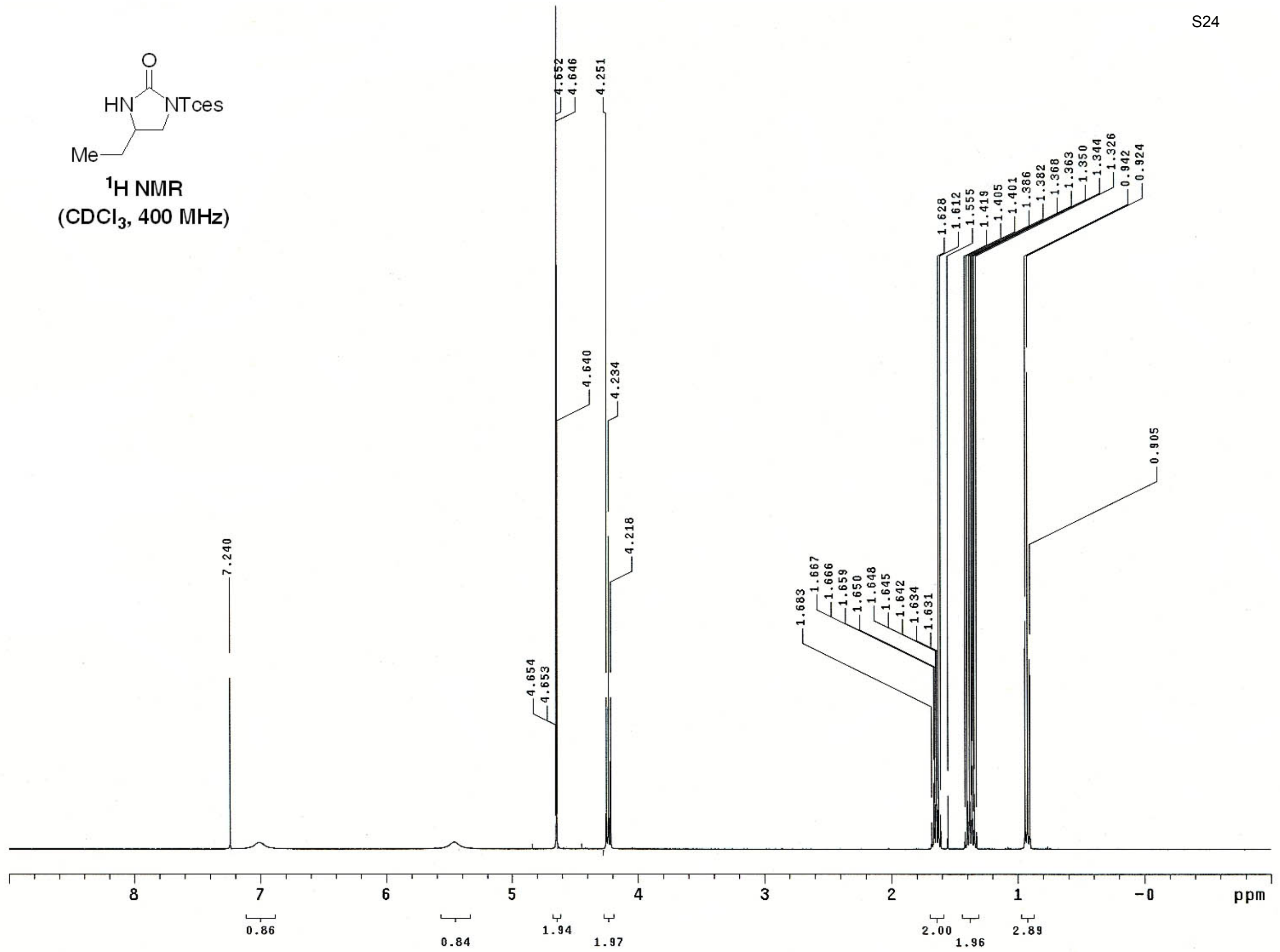




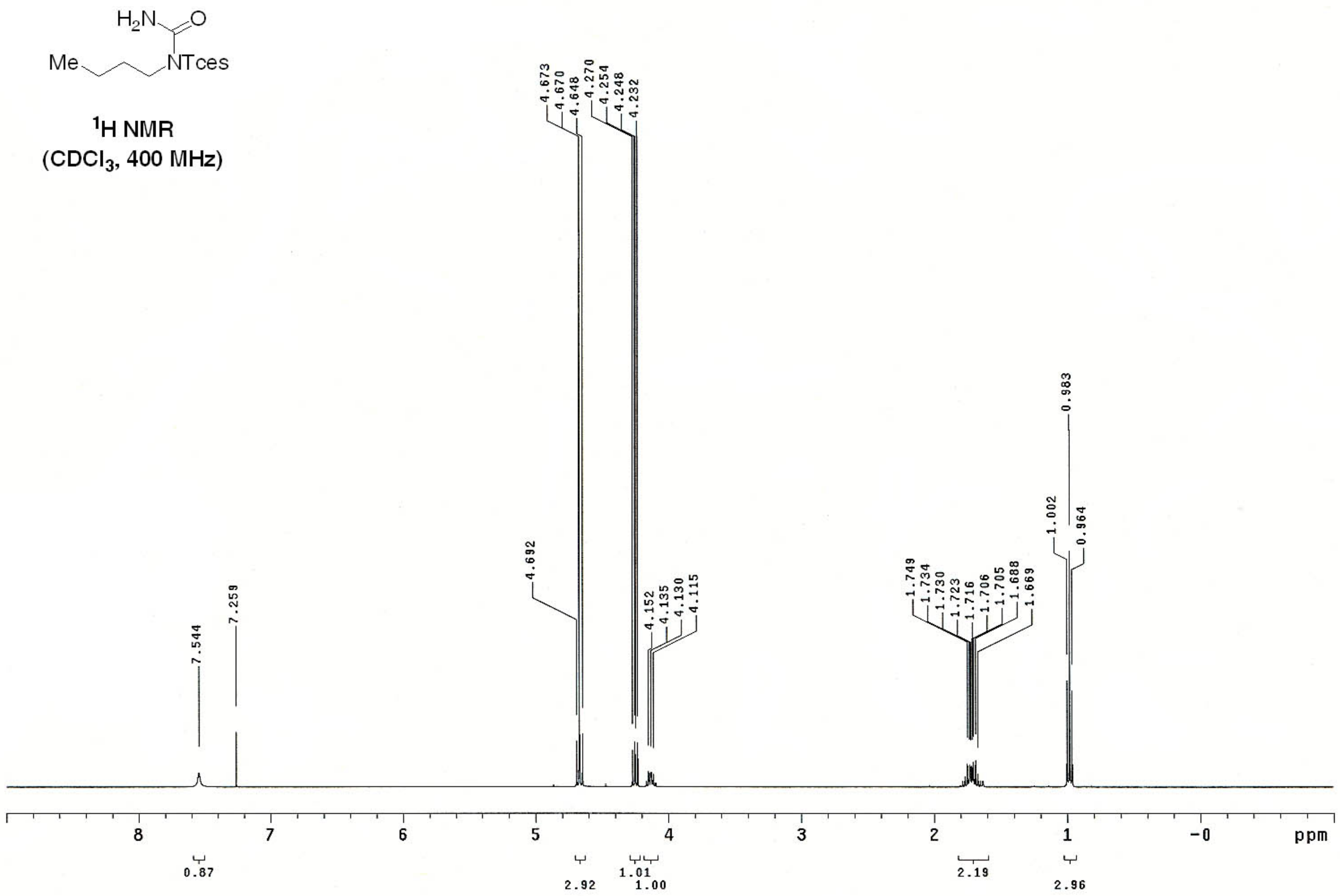


S26<smiles>CSC(C)=[N+]=C(C)C</smiles>

${ }^{1} \mathrm{H}$ NMR

$\left(\mathrm{CDCl}_{3}, 400 \mathrm{MHz}\right)$

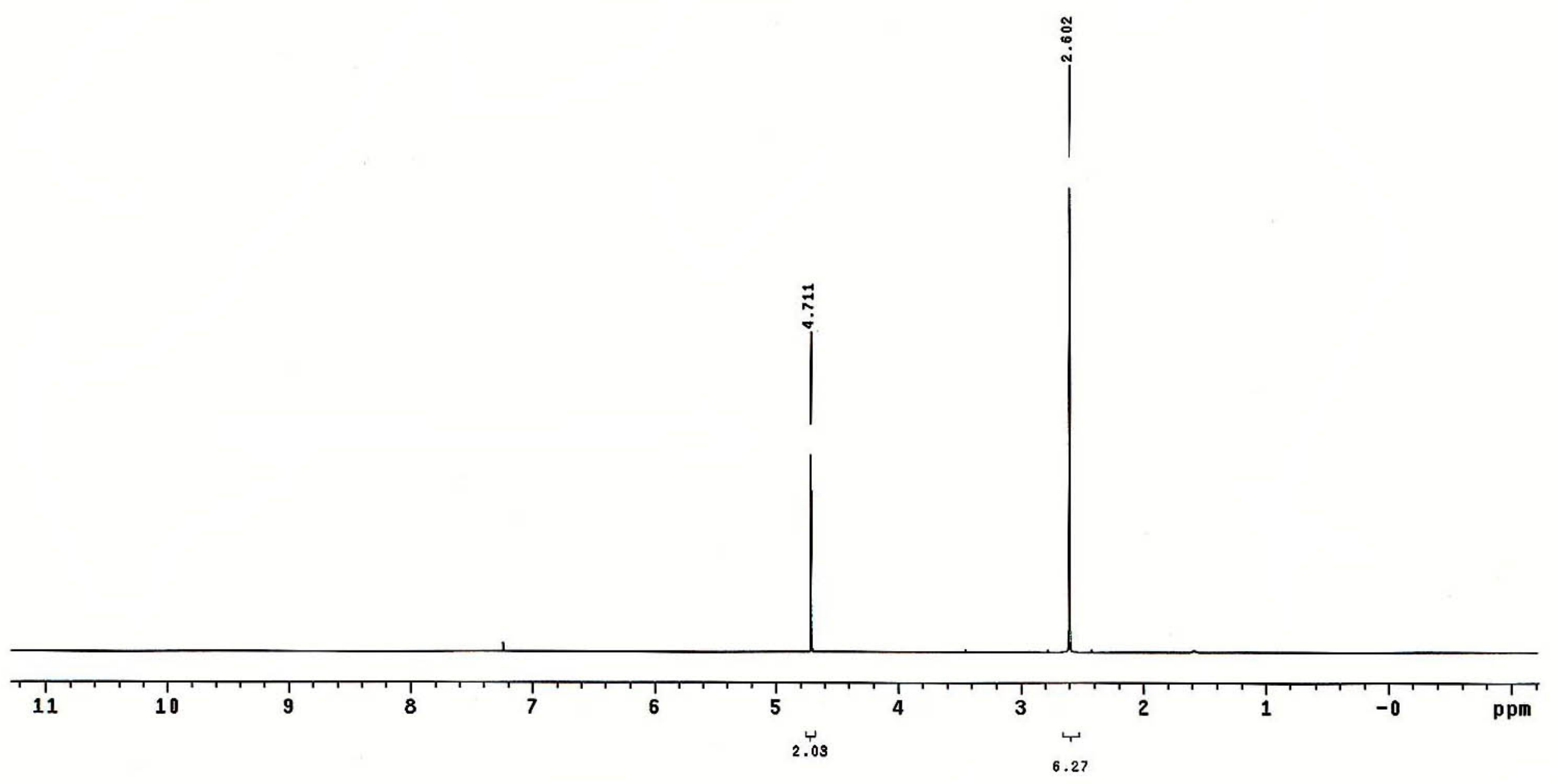




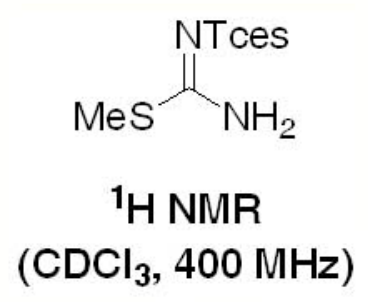

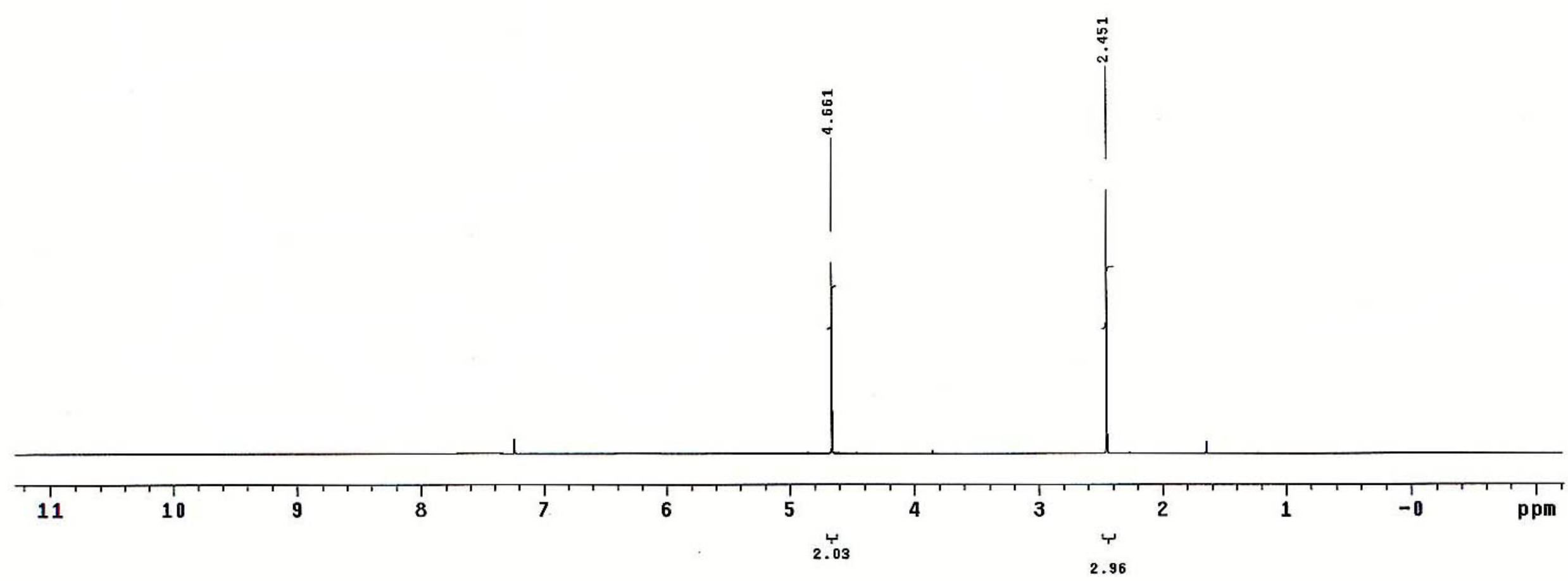




$$
\begin{gathered}
\text { NTces } \\
{ }^{1}{ }^{1} \mathrm{H} \text { NMR } \\
\left(\mathrm{CDCl}_{3}, 500 \mathrm{MHz}\right)
\end{gathered}
$$

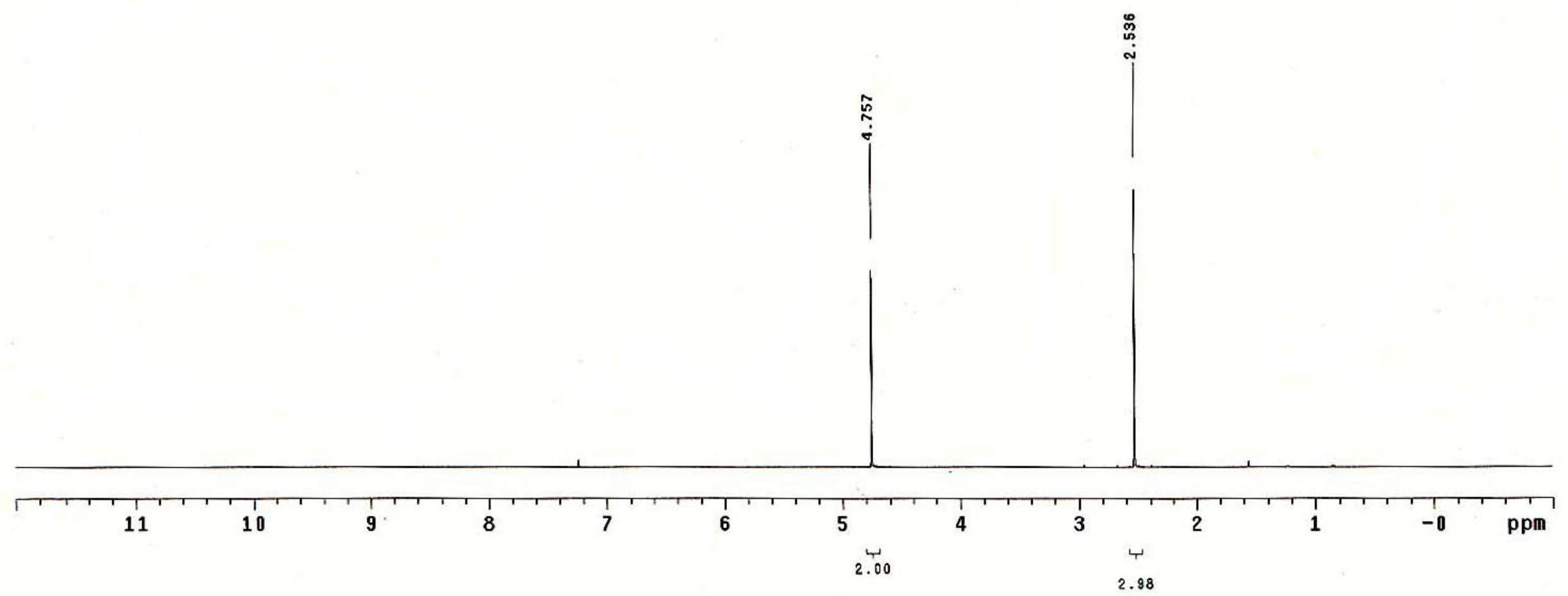



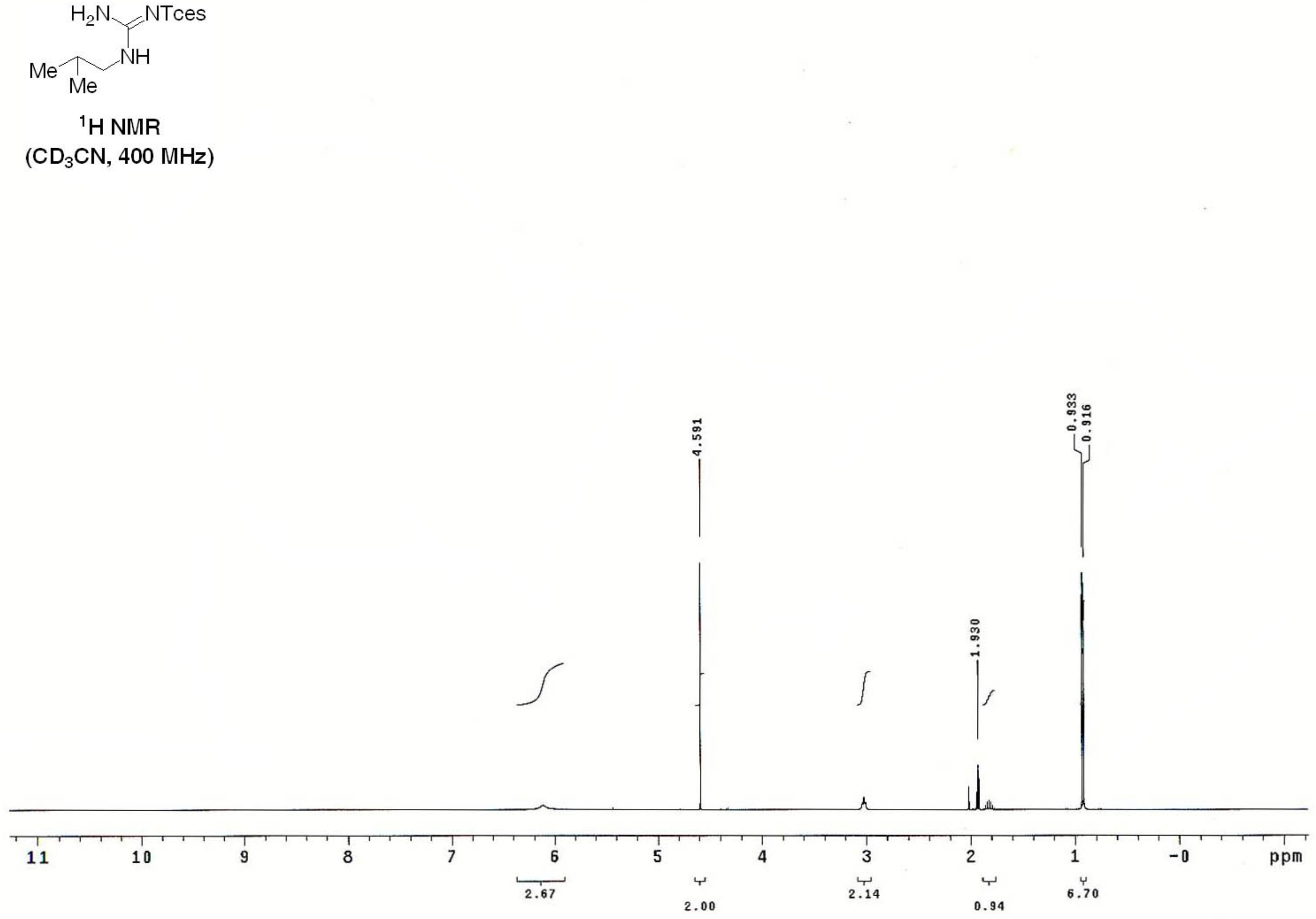


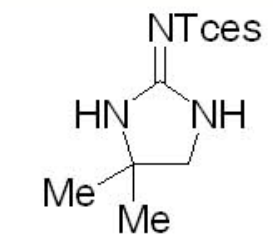

${ }^{1} \mathrm{H}$ NMR

$\left(\mathrm{CD}_{3} \mathrm{CN}, 400 \mathrm{MHz}\right)$

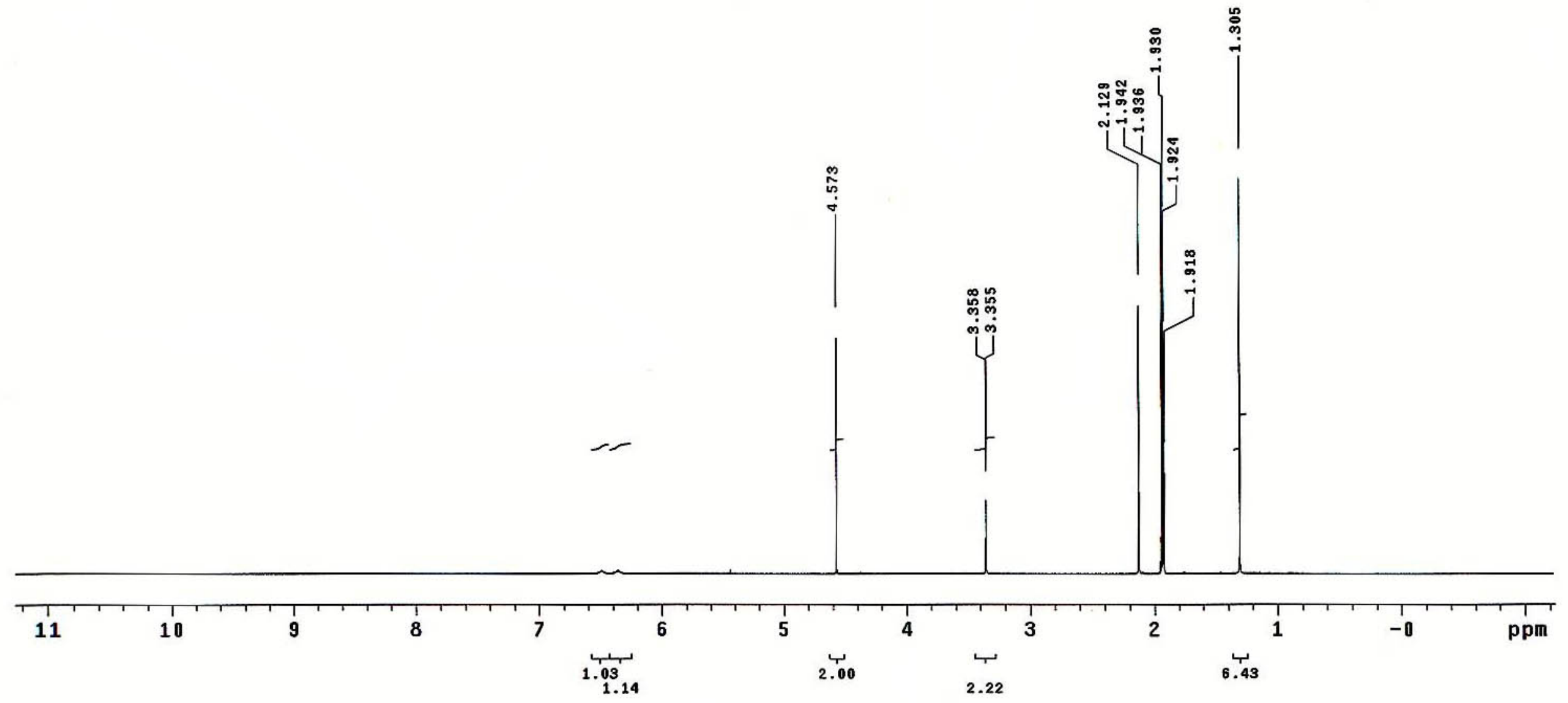




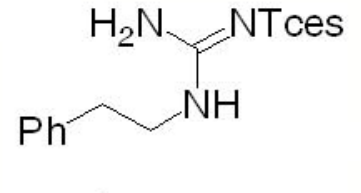

${ }^{1} \mathrm{H}$ NMR

$\left(\mathrm{CDCl}_{3}, 500 \mathrm{MHz}\right)$

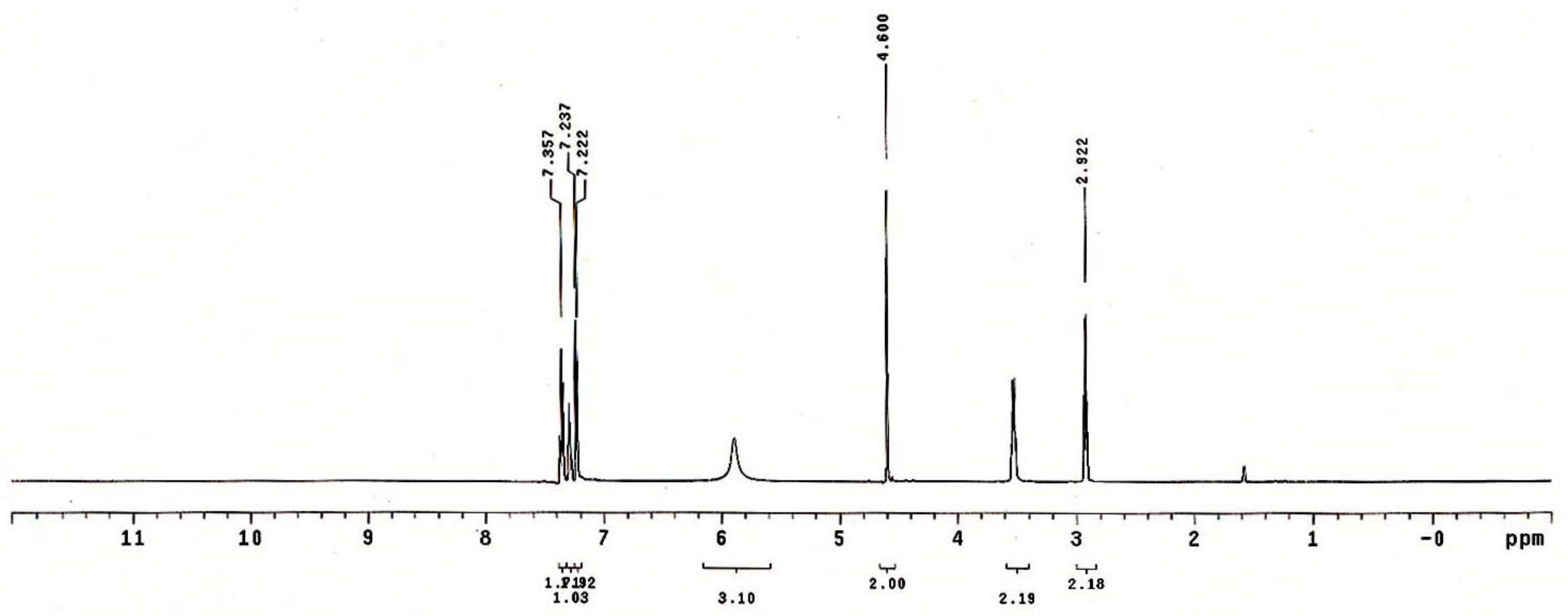




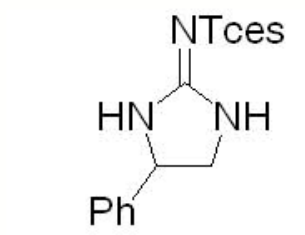

${ }^{1} \mathrm{H}$ NMR $\left(\mathrm{CD}_{3} \mathrm{CN}, 400 \mathrm{MHz}\right)$

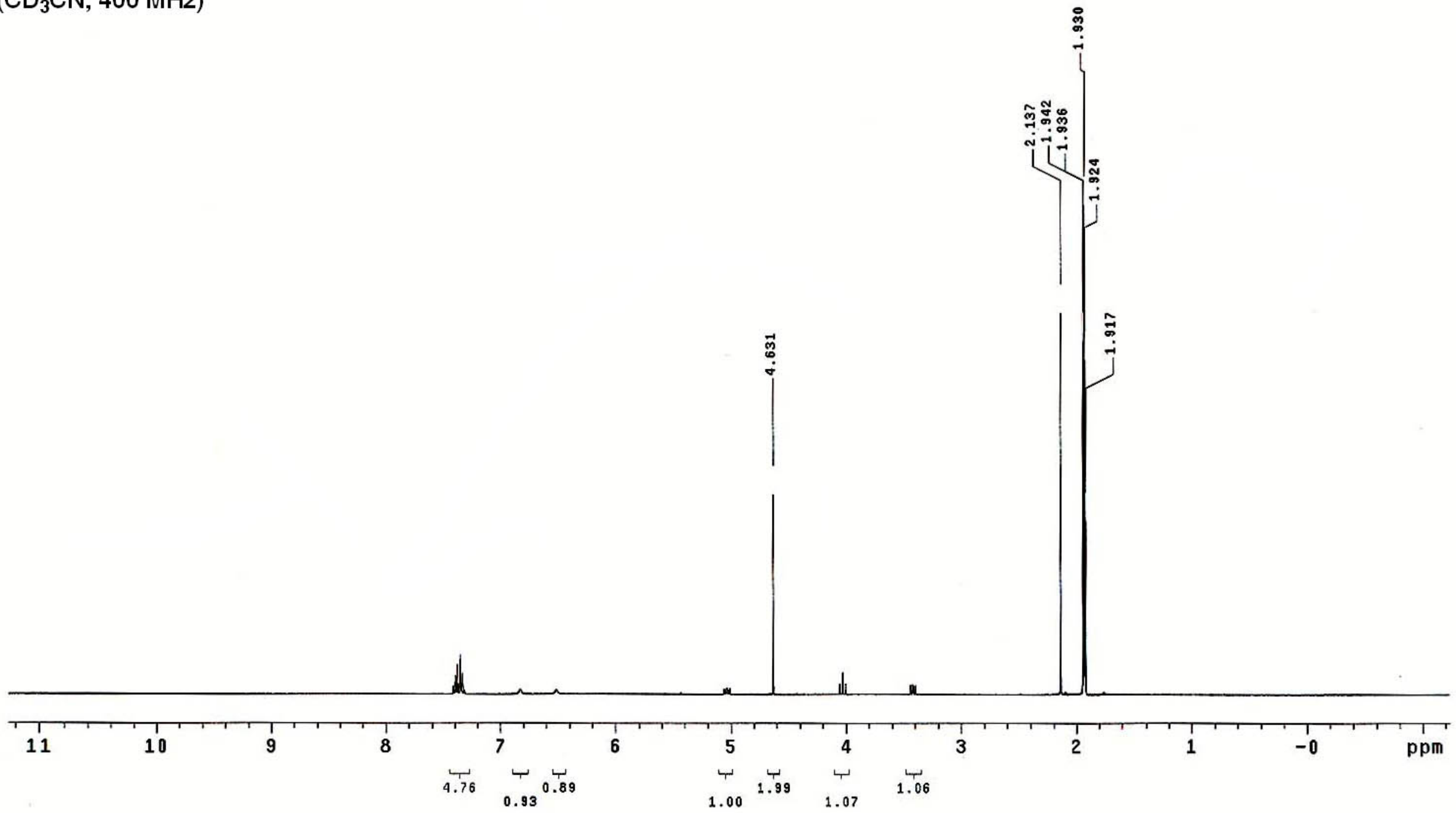




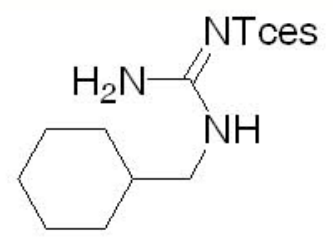

${ }^{1} \mathrm{H}$ NMR

$\left(\mathrm{CD}_{3} \mathrm{CN}, 400 \mathrm{MHz}, 60^{\circ} \mathrm{C}\right.$ )

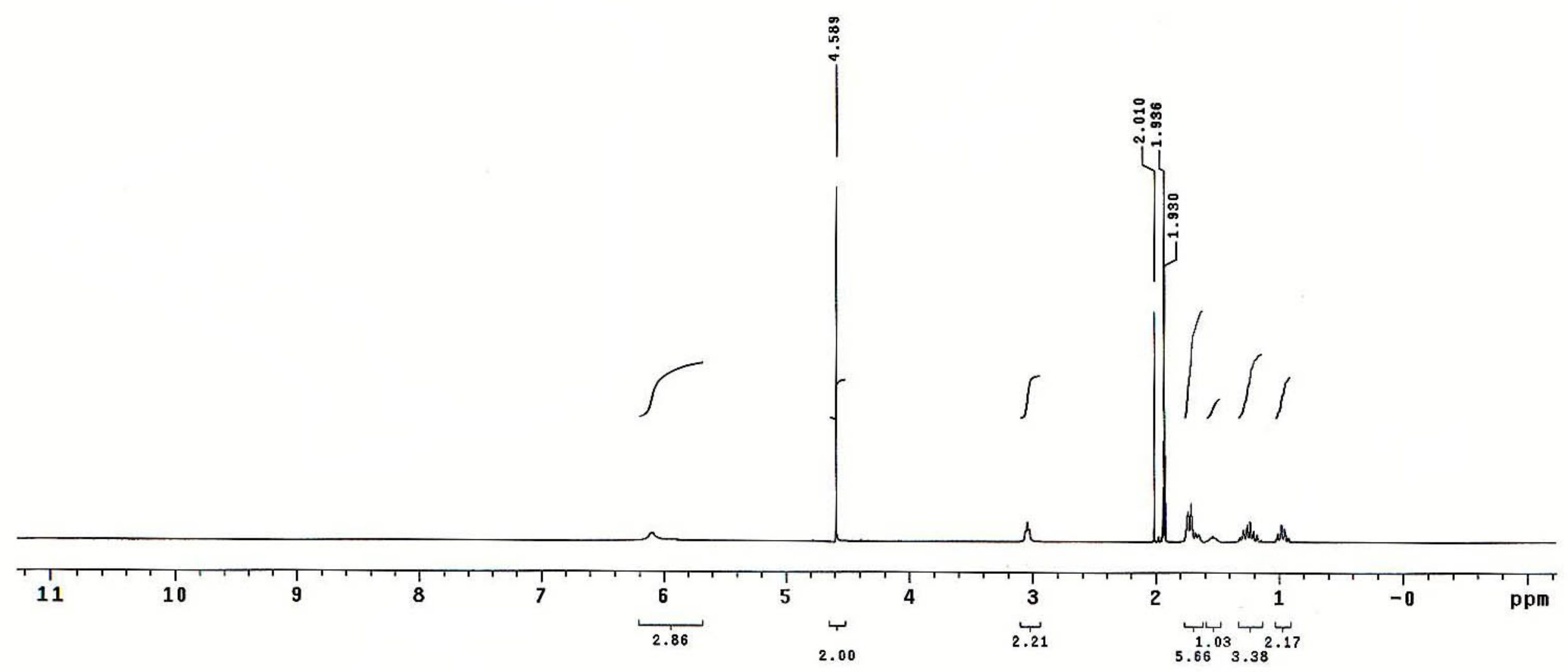




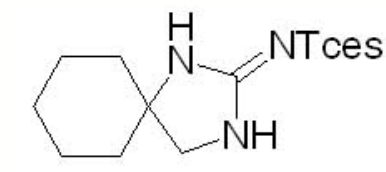

${ }^{1} \mathrm{H}$ NMR

$\left(\mathrm{CD}_{3} \mathrm{CN}, 400 \mathrm{MHz}\right)$

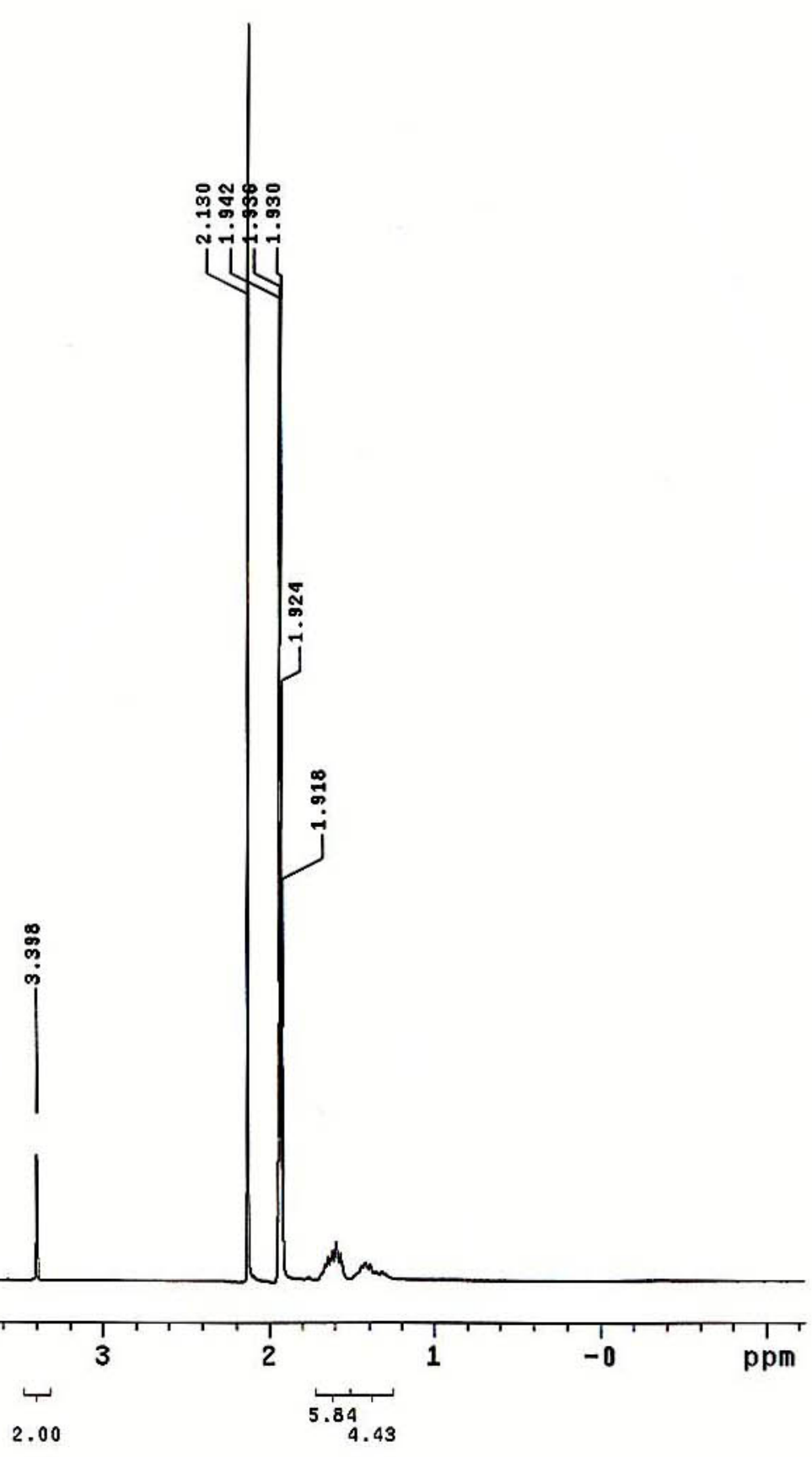


$\mathrm{H}_{2} \mathrm{~N} Y$ NTces

$\mathrm{Me}_{\mathrm{Me}}^{\mathrm{NH}}$

${ }^{1} \mathrm{H}$ NMR

$\left(\mathrm{CD}_{3} \mathrm{CN}, 400 \mathrm{MHz}, 65^{\circ} \mathrm{C}\right)$

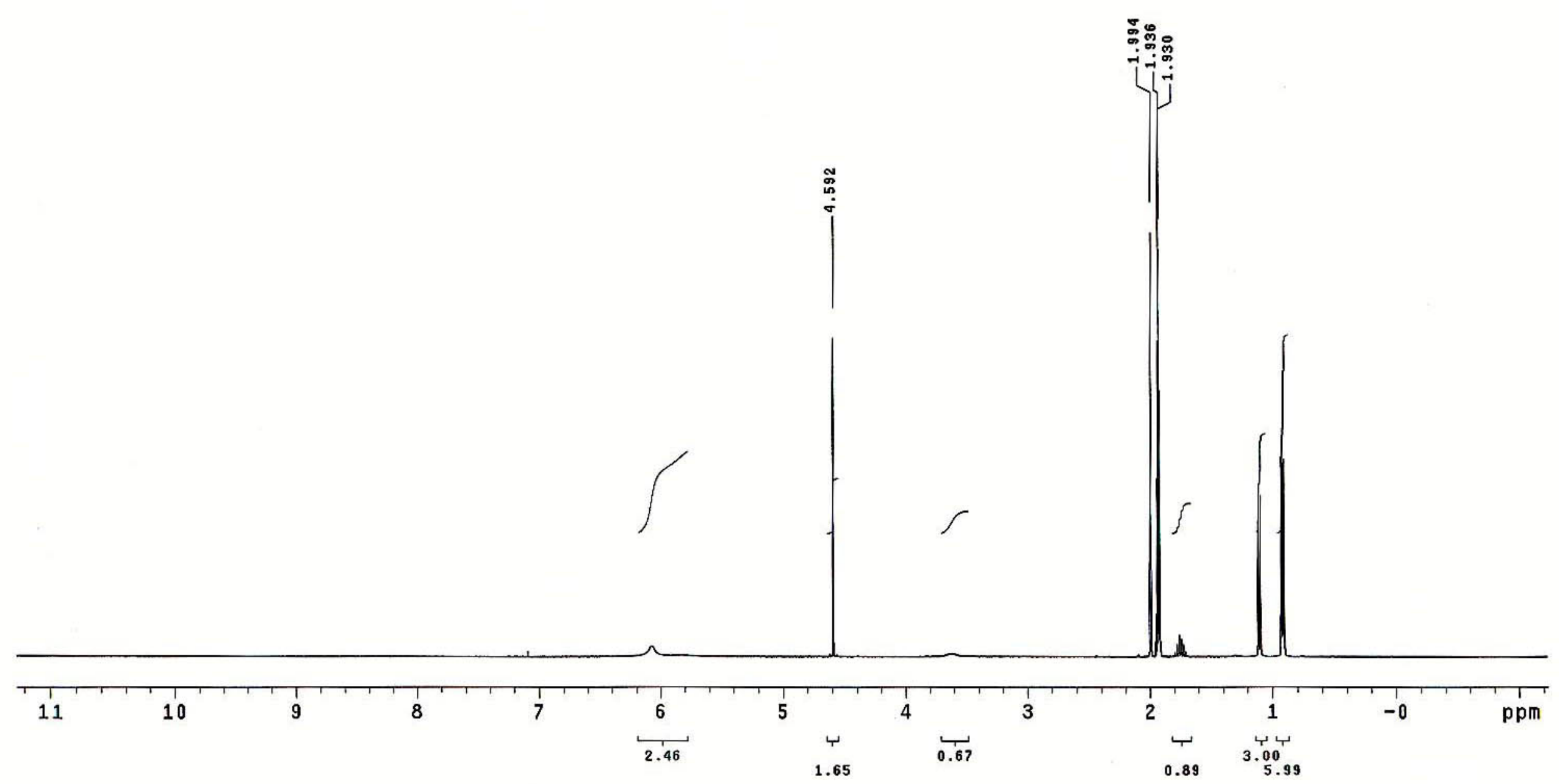




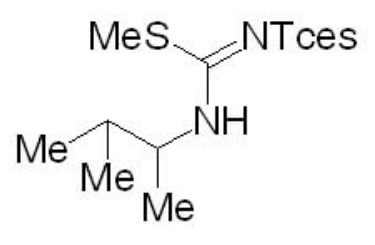

${ }^{1} \mathrm{H}$ NMR

$\left(\mathrm{CDCl}_{3}, 400 \mathrm{MHz}\right)$

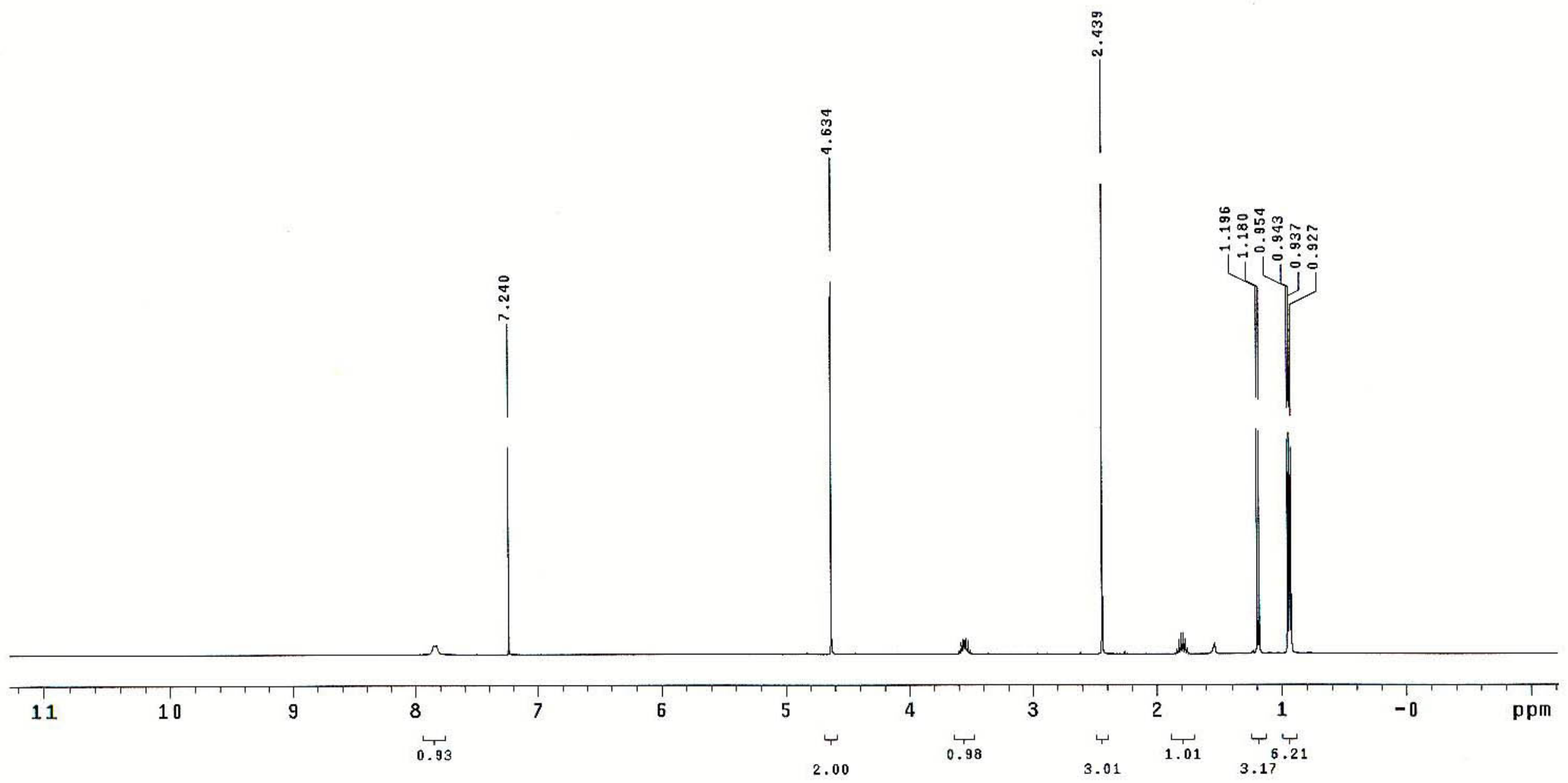




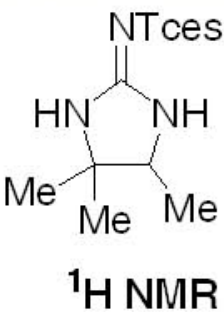

$\left(\mathrm{CDCl}_{3}, 500 \mathrm{MHz}, 50^{\circ} \mathrm{C}\right)$

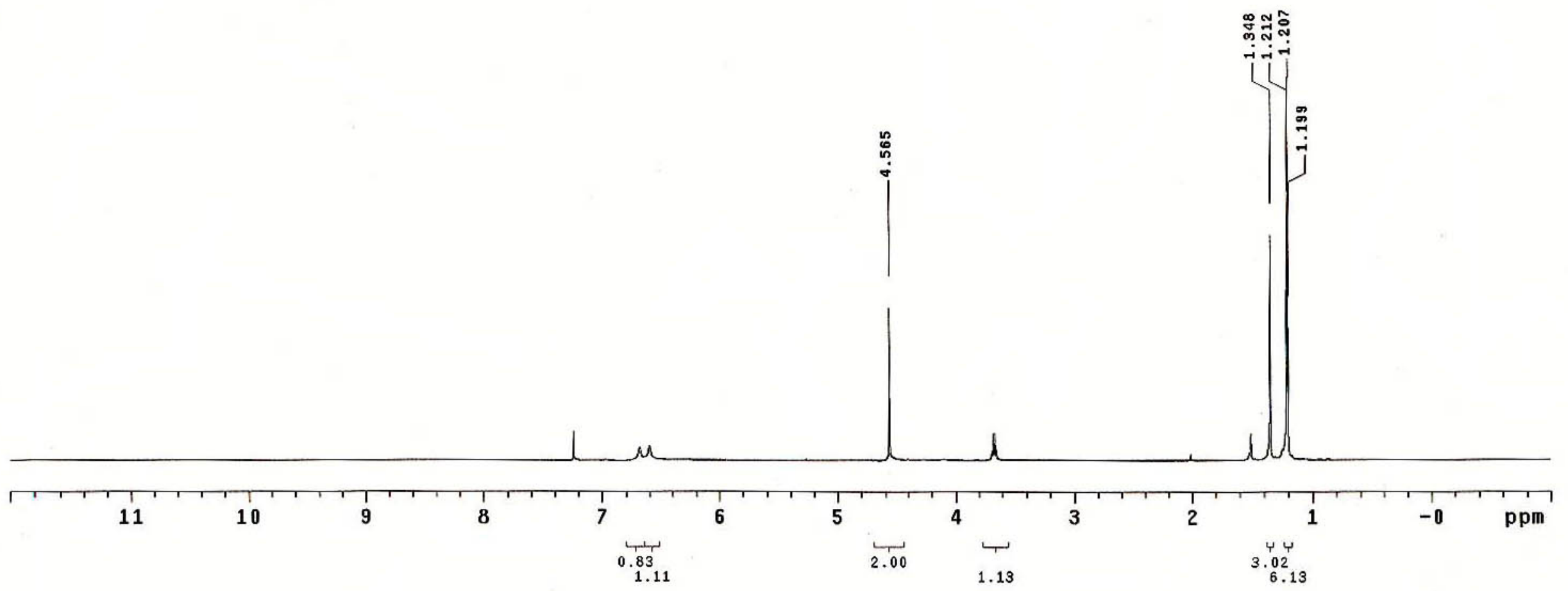




$$
\text { Ne }
$$$$
{ }^{1} \mathrm{H} \text { NMR }
$$

$\left(\mathrm{CD}_{3} \mathrm{CN}, 500 \mathrm{MHz}, 65^{\circ} \mathrm{C}\right)$

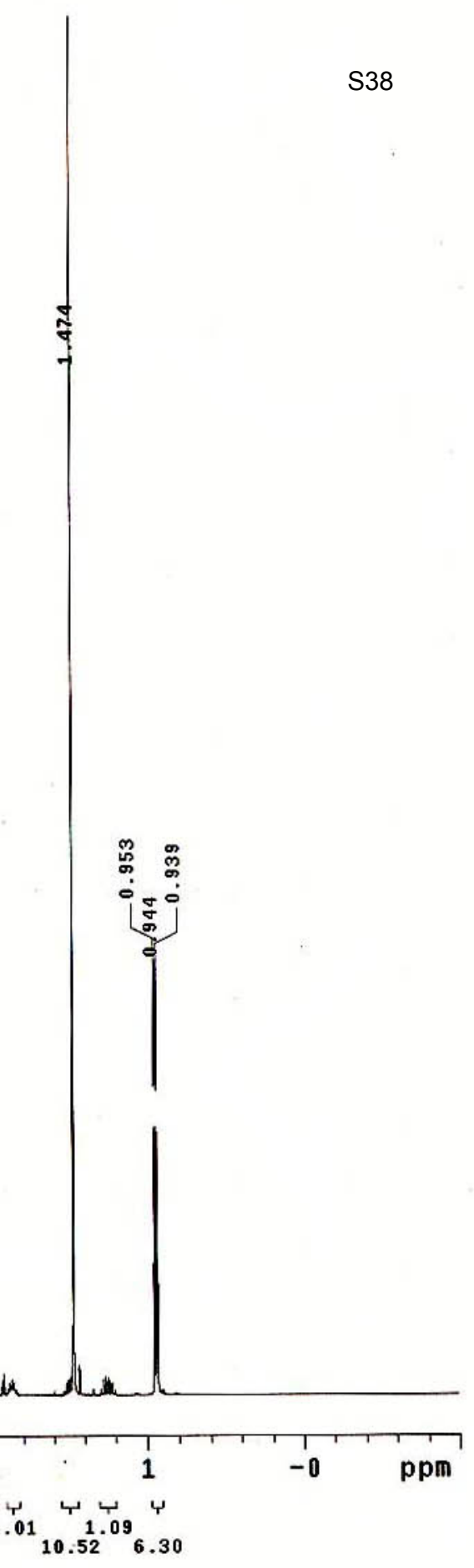




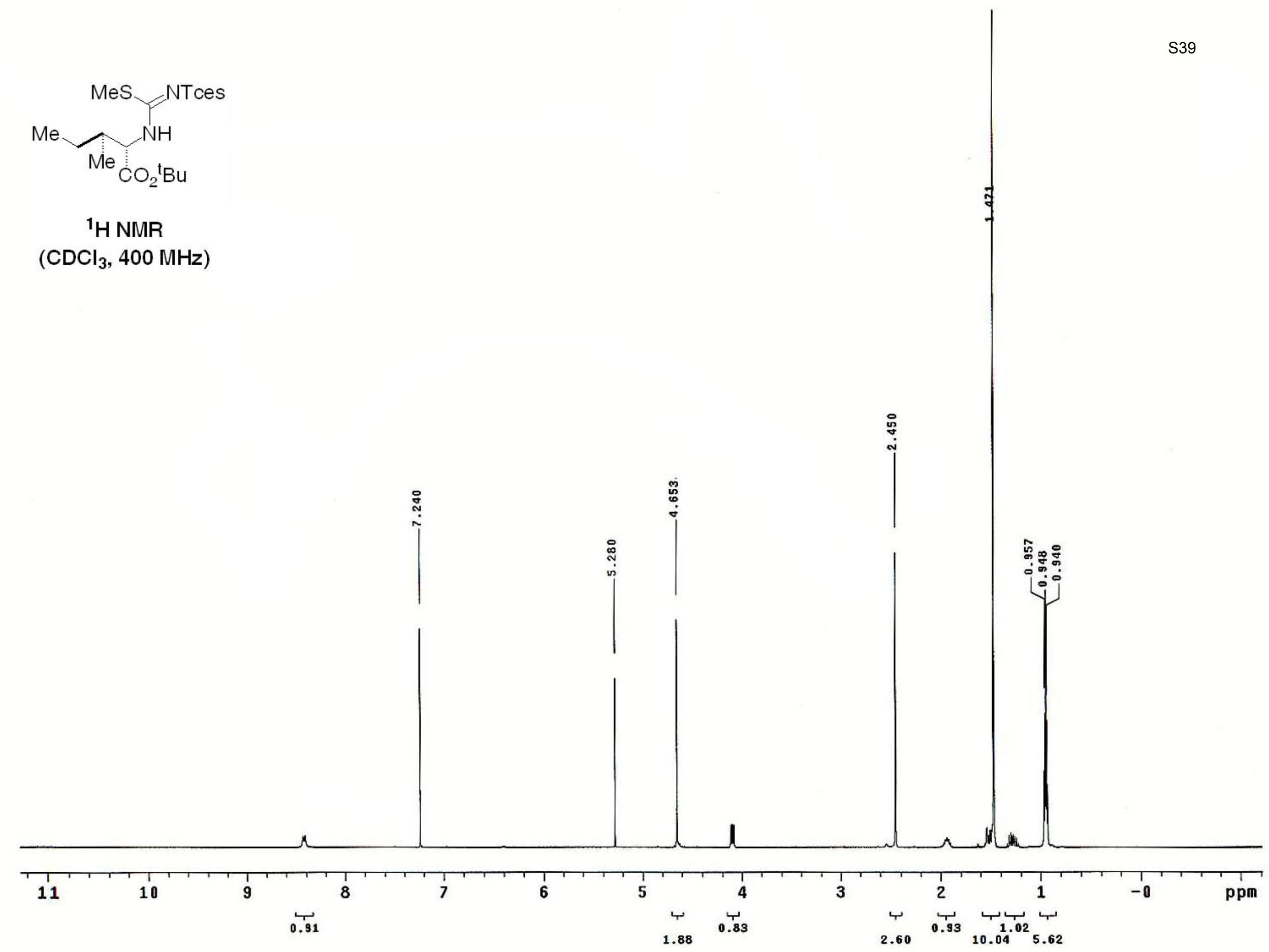




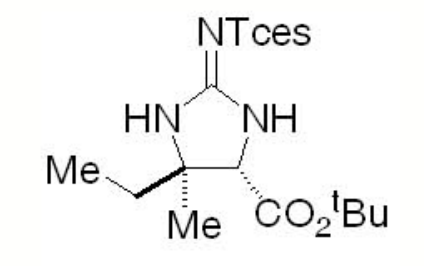

${ }^{1} \mathrm{H}$ NMR

$\left(\mathrm{CDCl}_{3}, 400 \mathrm{MHz}\right)$

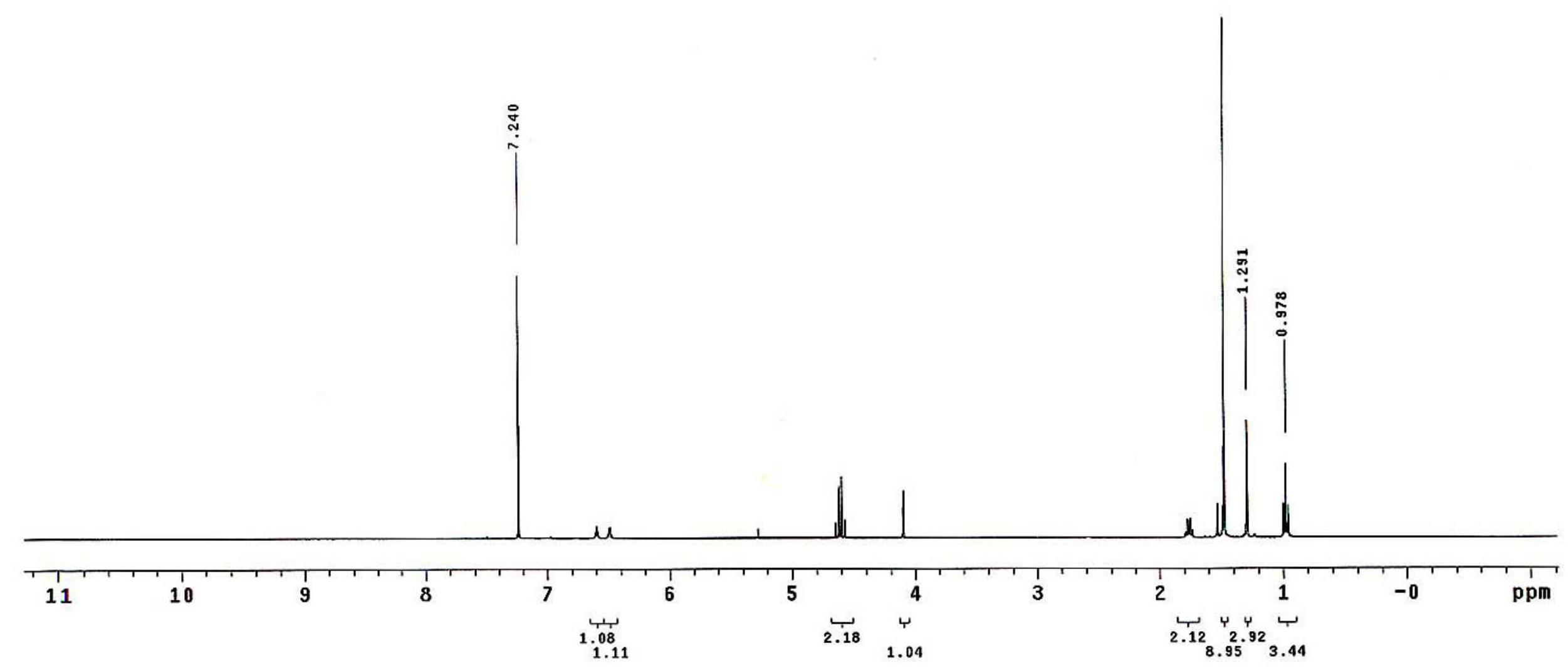




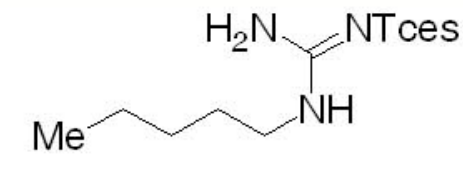

${ }^{1} \mathrm{H}$ NMR

$\left(\mathrm{CD}_{3} \mathrm{CN}, 400 \mathrm{MHz}, 70^{\circ} \mathrm{C}\right)$

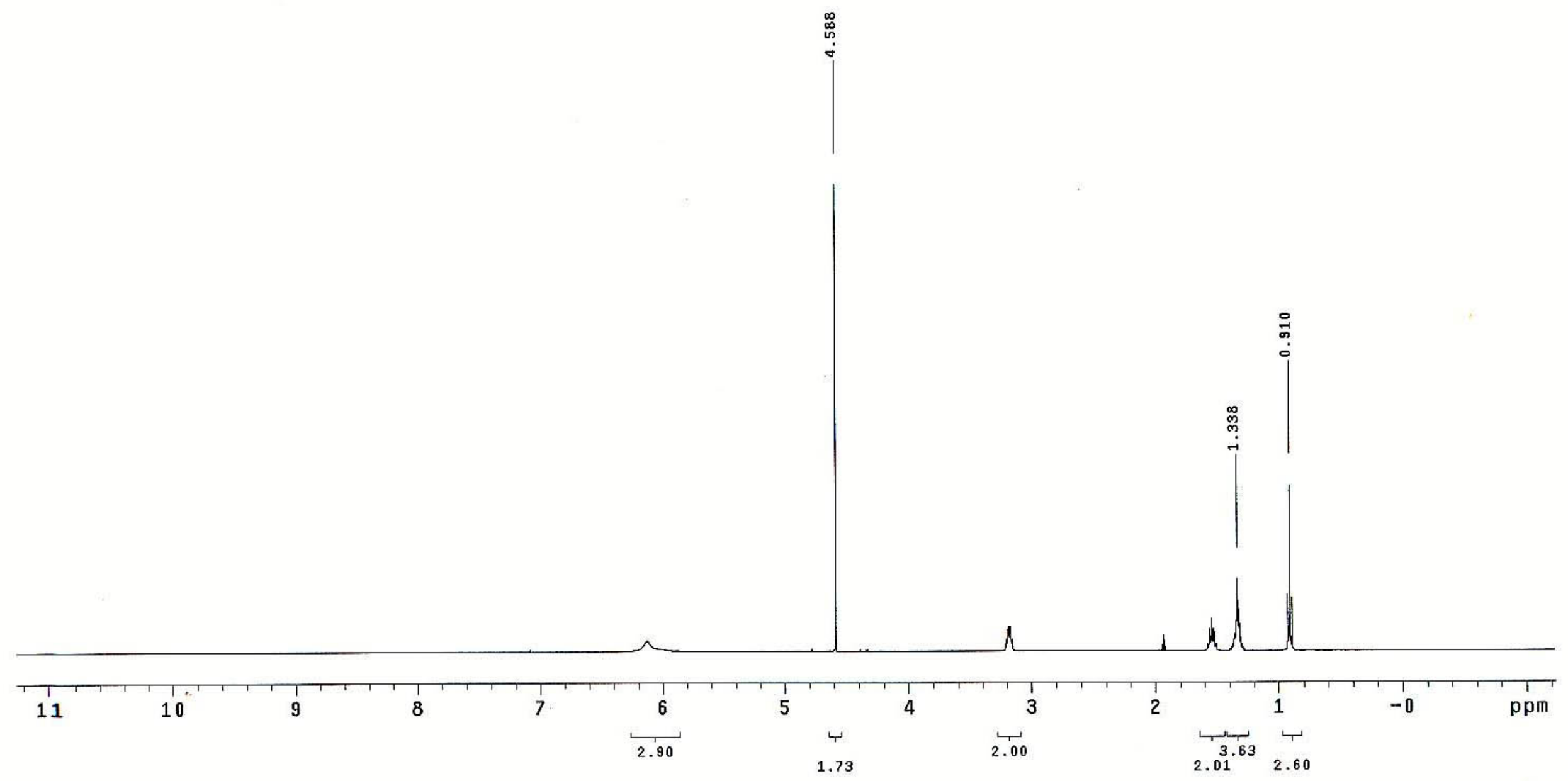




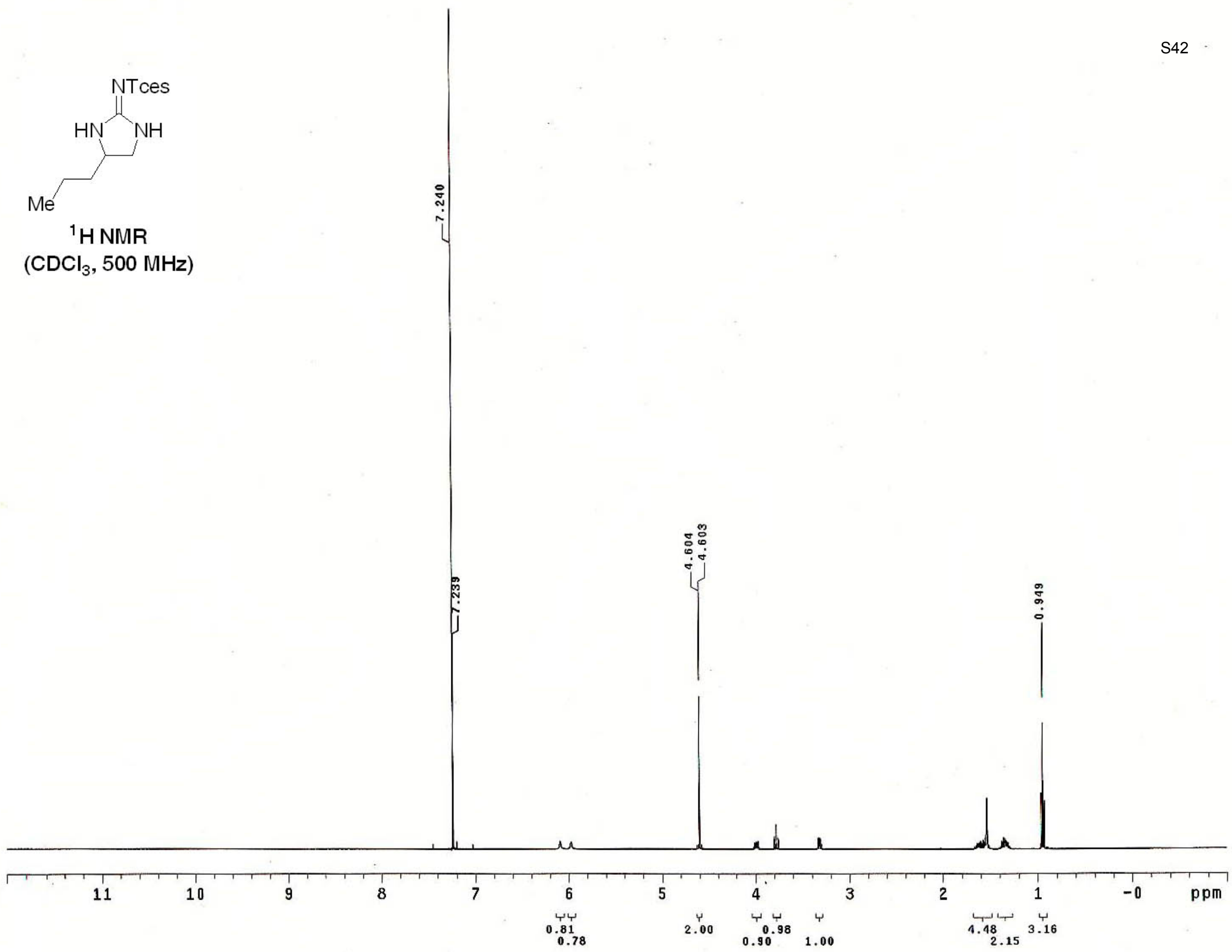


S43<smiles>[NH2+]=C1NCC(c2ccccc2)N1COC(=O)C(F)(F)F</smiles>

$$
\begin{gathered}
{ }^{1} \mathrm{H} \text { NMR } \\
\left(\mathrm{CD}_{3} \mathrm{OD}, 400 \mathrm{MHz}\right)
\end{gathered}
$$

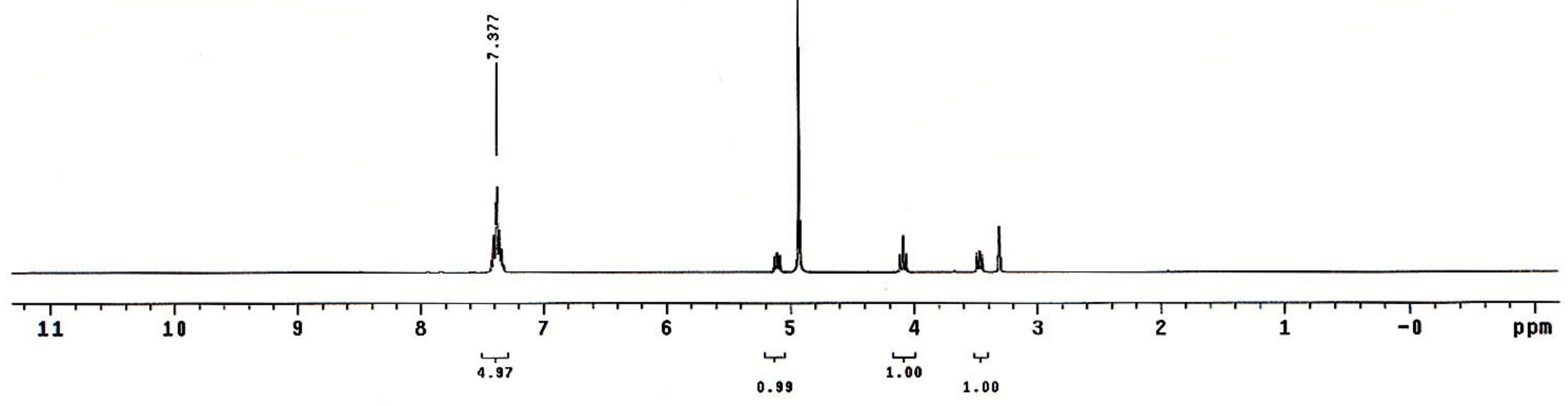

